\title{
Quantitative imaging of gas adsorption equilibrium and dynamics by X-ray computed tomography
}

\author{
Ronny Pini ${ }^{1}$ D $\cdot$ Lisa Joss $^{2} \cdot$ Sayed Alireza Hosseinzadeh Hejazi ${ }^{3}$
}

Received: 1 June 2020 / Revised: 3 September 2020 / Accepted: 10 September 2020 / Published online: 26 September 2020

(c) The Author(s) 2020

\begin{abstract}
We present the development and application of X-ray Computed Tomography (CT) for the determination of the adsorption properties of microporous adsorbents and the study of breakthrough experiments in a laboratory fixed-bed adsorption column. Using the model system $\mathrm{CO}_{2}$ /helium on activated carbon, equilibrium and dynamic adsorption/desorption measurements by $\mathrm{X}$-ray CT are described, and the results are successfully compared to those obtained from conventional methods, including the application of a one-dimensional dynamic column breakthrough model. The study demonstrates the practical feasibility of applying X-ray CT to measure internal and transient concentration profiles in adsorbent systems on the length-scales from a single adsorbent pellet to a packed column.
\end{abstract}

Keywords Breakthrough experiments $\cdot$ Adsorption imaging $\cdot$ Multi-scale tomography

\begin{tabular}{|c|c|}
\hline & iations \\
\hline$A$ & Column cross sectional area $\left[\mathrm{m}^{2}\right]$ \\
\hline$b$ & $\begin{array}{l}\text { Parameter in dual-site Langmuir isotherm } \\
{\left[\mathrm{m}^{3} \mathrm{~mol}^{-1}\right]}\end{array}$ \\
\hline$b_{0}$ & $\begin{array}{l}\text { Parameter in dual-site Langmuir isotherm } \\
{\left[\mathrm{m}^{3} \mathrm{~mol}^{-1}\right]}\end{array}$ \\
\hline$c$ & Gas phase concentration $\left[\mathrm{mol} \mathrm{m}^{-3}\right]$ \\
\hline$C_{\mathrm{p}, \mathrm{a}}$ & $\begin{array}{l}\text { Specific heat capacity of the adsorbed phase } \\
{\left[\mathrm{J} \mathrm{mol}^{-1} \mathrm{~K}^{-1}\right]}\end{array}$ \\
\hline$c_{p, g}$ & Specific heat capacity of the gas phase $\left[\mathrm{J} \mathrm{mol}^{-1} \mathrm{~K}^{-1}\right]$ \\
\hline$C_{\mathrm{p}, \mathrm{s}}$ & Specific heat capacity of the adsorbent $\left[\mathrm{J} \mathrm{kg}^{-1} \mathrm{~K}^{-1}\right]$ \\
\hline$C_{\mathrm{p}, \mathrm{w}}$ & $\begin{array}{l}\text { Specific heat capacity of the column wall } \\
{\left[\mathrm{J} \mathrm{kg}^{-1} \mathrm{~K}^{-1}\right]}\end{array}$ \\
\hline$C T$ & $\mathrm{CT}$ number $[\mathrm{HU}]$ \\
\hline$D_{\mathrm{L}}$ & Axial dispersion $\left[\mathrm{m}^{2} \mathrm{~s}^{-1}\right]$ \\
\hline
\end{tabular}

Electronic supplementary material The online version of this article (https://doi.org/10.1007/s10450-020-00268-7) contains supplementary material, which is available to authorized users.

Ronny Pini

r.pini@imperial.ac.uk

1 Department of Chemical Engineering, Imperial College London, London SW7 2AZ, UK

2 School of Chemical Engineering and Analytical Science, The University of Manchester, Manchester, UK

3 Department of Chemical Engineering, Amirkabir University of Technology (Tehran Polytechnic), Tehran, Islamic Republic of Iran
$D_{\mathrm{m}} \quad$ Molecular diffusivity $\left[\mathrm{m}^{2} \mathrm{~s}^{-1}\right]$

$f \quad$ Flow rate $\left[\mathrm{m}^{3} \mathrm{~min}^{-1}\right]$

$H^{\text {ex }} \quad$ Hounsfield units of the excess amount adsorbed per voxel volume [HU]

$h_{\text {in }} \quad$ Inside heat transfer coefficient $\left[\mathrm{J} \mathrm{m}^{-2} \mathrm{~K}^{-1} \mathrm{~s}^{-1}\right]$

$h_{\text {out }}$ Outside heat transfer coefficient $\left[\mathrm{J} \mathrm{m}^{-2} \mathrm{~K}^{-1} \mathrm{~s}^{-1}\right]$

$J \quad$ Minimization objective function

$k_{\mathrm{i}} \quad$ Mass transfer coefficient $\left[\mathrm{s}^{-1}\right]$

$K_{\mathrm{w}} \quad$ Thermal conductivity of column wall $\left[\mathrm{J} \mathrm{m}^{-1} \mathrm{~K}^{-1} \mathrm{~s}^{-1}\right]$

$K_{z} \quad$ Effective gas thermal conductivity $\left[\mathrm{J} \mathrm{m}^{-1} \mathrm{~K}^{-1} \mathrm{~s}^{-1}\right]$

$L \quad$ Column length $[\mathrm{m}]$

$m^{\text {ex }} \quad$ Excess amount adsorbed per voxel volume $\left[\mathrm{kg} \mathrm{m}^{-3}\right]$

$M_{\mathrm{m}} \quad$ Molecular mass $\left[\mathrm{kg} \mathrm{mol}^{-1}\right]$

$N \quad$ Number of time steps

$p \quad$ Pressure $[\mathrm{Pa}]$

$p^{*} \quad$ Reference pressure $[\mathrm{Pa}]$

$p_{1} \quad$ CT number calibration coefficient $\left[\mathrm{HU} \mathrm{m}^{3} \mathrm{~kg}^{-1}\right]$

$p_{2} \quad$ CT number calibration coefficient $\left[\mathrm{HU} \mathrm{m}^{3} \mathrm{~kg}^{-1}\right]$

$q \quad$ Solid phase concentration $\left[\mathrm{mol} \mathrm{kg}^{-1}\right.$ ]

$q_{s} \quad$ Saturation concentration in the solid phase $\left[\mathrm{mol} \mathrm{kg}^{-1}\right.$ ]

$q^{*} \quad$ Equilibrium solid phase concentration $\left[\mathrm{mol} \mathrm{kg}^{-1}\right.$ ]

$R \quad$ Universal gas constant $\left[\mathrm{Pa} \mathrm{m}^{3} \mathrm{~mol}^{-1} \mathrm{~K}^{-1}\right]$

$r_{\text {in }} \quad$ Column inner radius [m]

$r_{\text {out }}$ Column outer radius [m]

$r_{\mathrm{p}} \quad$ Particle radius [m]

$t \quad$ Time [s]

$T$ Temperature [K]

$T_{\mathrm{a}} \quad$ Ambient temperature [K] 
$t_{\mathrm{bt}} \quad$ Breakthrough time [s]

$t_{\mathrm{r}} \quad$ Residence time [s]

$T_{\mathrm{w}} \quad$ Column wall temperature $[\mathrm{K}]$

$T^{*} \quad$ Reference temperature $[\mathrm{K}]$

$U \quad$ Internal energy $\left[\mathrm{J} \mathrm{mol}^{-1}\right]$

$v \quad$ Interstitial velocity $\left[\mathrm{m} \mathrm{s}^{-1}\right]$

$V \quad$ Volume $\left[\mathrm{m}^{3}\right]$

$V_{\mathrm{b}} \quad$ Bed volume $\left[\mathrm{m}^{3}\right]$

$v_{\mathrm{m}} \quad$ Specific micropore volume $\left[\mathrm{m}^{3} \mathrm{~kg}^{-1}\right]$

$v_{\mathrm{sh}} \quad$ Shock front velocity $\left[\mathrm{m} \mathrm{s}^{-1}\right]$

$y \quad$ Gas phase composition

$z \quad$ Column internal location [m]

\section{Greek symbols}

$\epsilon \quad$ bed voidage

$\epsilon_{\mathrm{p}} \quad$ Particle voidage

$\epsilon_{\mathrm{t}} \quad$ Total porosity of a solid

$\mu \quad$ Fluid viscosity $\left[\mathrm{kg} \mathrm{m}^{-1} \mathrm{~s}^{-1}\right]$

$\rho \quad$ Bulk density $\left[\mathrm{kg} \mathrm{m}^{-3}\right]$

$\rho_{\mathrm{b}} \quad$ Bed density $\left[\mathrm{kg} \mathrm{m}^{-3}\right]$

$\rho_{\mathrm{L}} \quad$ Adsorbed phase density $\left[\mathrm{kg} \mathrm{m}^{-3}\right]$

$\rho_{\mathrm{p}} \quad$ Adsorbent particle density $\left[\mathrm{kg} \mathrm{m}^{-3}\right]$

$\rho_{\text {sk }} \quad$ Adsorbent skeleton density $\left[\mathrm{kg} \mathrm{m}^{-3}\right]$

$\rho_{\mathrm{w}} \quad$ Column wall density $\left[\mathrm{kg} \mathrm{m}^{-3}\right]$

$\tau \quad$ Tortuosity

\section{Introduction}

Large-scale gas adsorption separations are commonly carried out as cyclic processes, in which the adsorbent bed undergoes alternately saturation and regeneration. Because they enable independent verification of each individual step in the cyclic process, dynamic column experiments are fundamental to the design of adsorption-based separations. Traditionally, these experiments are carried out with a packed adsorption column by acquiring transient data for the outlet gas composition and volumetric flow rate, as well as for the temperature measured at one or more locations within the adsorbent column. The one-dimensional axially dispersed plug flow model is commonly applied to simulate the experiments by solving material balances for the fluid and adsorbed phase within the adsorption column, together with the relevant energy balances (Ruthven 1984). The numerical simulation is not only used to verify the validity of experimental data measured independently (e.g., equilibrium isotherms or kinetic coefficients), but also to obtain additional process-relevant parameters, such as hydrodynamic dispersion as well as heat and mass transfer coefficients of the adsorption system under consideration.

Because it relies on measurements acquired at a specific location (i.e. the outlet of the column), the approach described above necessarily provides average properties, thereby yielding parameter values that may lump effects arising from localised phenomena within the adsorbent column (Knox James et al. 2016). Deviations from the expected averaged behaviour may be due to the inherent variability of the size, shape and composition of the formulated adsorbent particles (Nepryahin et al. 2016; Martin et al. 2017); sorbent deactivation (Cheah et al. 1994); packing heterogeneities (Johnson et al. 2017); and flow maldistribution (Kwapinski et al. 2004; Sharma et al. 2020). Quite possibly, the two parameters that are mostly affected by these physical non-idealities are the mass and heat transfer coefficients as well as the axial dispersion coefficient, as these are often used as adjustable parameters to match the experimental breakthrough curves (Knox James et al. 2016). In this context, knowledge of the bed internal concentration profiles would not only provide a means to verify the experiment design, but also to improve the robustness of the modelling approach and the reliability of the obtained parameters. More advanced adsorber designs, such as those using layered beds (Sircar and Golden 2006) or structured adsorbents (DeWitt et al. 2018; Sharma et al. 2020) provide for an additional level of complexity in the design of the separation process. While it is now computationally feasible to model cyclic adsorption processes in 3D with computational fluid dynamics approaches (Gautier et al. 2018; Lian et al. 2019; Qasem and Ben-Mansour 2018), such models have so far mostly been applied to cylindrical homogeneous packings; their extension would enable the description of contactor geometries that cannot be captured by traditional onedimensional models (Claessens et al. 2020; Sharma et al. 2020; Wurzbacher et al. 2016). These advanced numerical approaches would greatly benefit from the development of experimental methods that enable the direct measurement of transient concentration profiles in adsorbent columns.

Experimental approaches that enable in-situ imaging of concentration profiles within adsorbent columns have been proposed over the last few decades, although these have only been marginally applied. Positron emission tomography (PET) (Anderson et al. 1998; Noordhoek et al. 1998) and magnetic resonance imaging (MRI) (Bär et al. 2002; Cheng et al. 2005; Pavlovskaya et al. 2015) have been successfully deployed, as both methods provide high temporal resolution and enable probing adsorption dynamics on the scale of a laboratory adsorption column. As recently reviewed in Pini and Joss (2019), the two approaches present notable features for the study of adsorption systems, including suitability to both gas and liquid systems and high molecule-specific sensitivity. So far, quantitative studies have been largely limited to one-dimension only, although both MRI and PET are designed to enable the three-dimensional visualisation of the sampled data-set. More recently, X-ray Computed Tomography (CT) was applied to measure in three-dimensions gas adsorption isotherms in microporous adsorbents 
and their packings (Joss and Pini 2017, 2019), but attempts to image adsorption dynamics have so far been only qualitative (Lodewyckx et al. 2006). X-ray CT is highly versatile and the technique enables probing a wide range of object sizes and spatial resolutions, from $\mathrm{mm}$-sized samples imaged at micron-resolution using commercial desktop scanners to metre-sized samples imaged at mm-resolution using medical scanners.

In this study, we demonstrate the applicability of X-ray CT to the study of adsorption dynamics in a laboratory adsorbent column at both particle and column scales. To this end, the model system $\mathrm{CO}_{2}$ /helium on activated carbon is chosen to evaluate its equilibrium and dynamic adsorption/desorption properties from breakthrough column experiments combined with simultaneous imaging of the internal transient concentration profiles by X-ray CT. The results are compared to those obtained independently from conventional methods, including the application of a one-dimensional dynamic column breakthrough model. We will briefly review in the following the basic principles of $\mathrm{X}$-ray CT with specific reference to the study of adsorption systems.

\section{Theory}

We will intentionally limit our presentation to the use of medical X-ray CT scanners by describing their basic measurement principles. For the interested reader, reviews are available in the literature that cover the full spectrum of quantitative X-ray CT methods (e.g., Maire and Whiters 2014).

The quantity measured in X-ray CT is the linear attenuation coefficient, $\mu$, which by means of Beer's law relates the intensity of the incident $X$-ray to the value remaining after it has passed through a dense object (Vinegar and Wellington 1987). To obtain a three-dimensional reconstruction of an object, modern medical X-ray CT scanners rotate the X-ray source in a helical path around it and use a ring of detectors to measure the intensity of the transmitted X-ray. This enables the acquisition of a series of one-dimensional projections of X-ray attenuation at various angles through a slice of the object that the instrument processes mathematically to generate cross-sectional images of attenuation coefficients (the tomogram). The three-dimensional image of the object is obtained upon stacking together many tomograms. For medical X-ray CT scanners, such as the one used in this study, the measured attenuation coefficients are usually provided and reported as CT numbers in Hounsfield unit (HU). This is achieved through a simple linear transformation of the mass attenuation coefficient, such that distilled water and air take values of $0 \mathrm{HU}$ and $-1000 \mathrm{HU}$, respectively (Vinegar and Wellington 1987; Withjack 1988). For these instruments, X-ray energies above $100 \mathrm{kV}$ are used and the linear attenuation coefficient, or the CT number, is linearly proportional to the bulk density (Wellington and Vinegar 1987):

$C T=p_{1} \rho+p_{2}$

For a calibration that uses water and air, it follows that $p_{1}=1 \mathrm{HU} \mathrm{m}^{3} / \mathrm{kg}$ and $p_{2}=-1000 \mathrm{HU}$, but ad-hoc calibrations can also be carried out (Wellington and Vinegar 1987; Joss and Pini 2017). The key practical implication of Eq. 1 is that the CT number in any given voxel of the imaged object can be expressed as the linear combination of the attenuation from each of its components. Therefore, for systems involving multiple fluid phases in a porous solid, measurement interpretation is conveniently carried out on difference images that are obtained upon subtracting a properly registered reference image acquired at e.g. saturation with an inert gas, such as helium. As such, the dense solid is effectively removed from the reconstructed image and fluid saturation can be computed by linear interpolation within the bounds of the calibration line (Withjack 1988; Pini 2014).

For a porous solid (s) exposed to an adsorptive gas (a) at pressure $p$ and temperature $T$, the following expression for the measured local CT number is obtained (Pini 2014):

$\overline{C T}_{\mathrm{a}}(p, T)=\epsilon_{\mathrm{t}} C T_{\mathrm{a}}(p, T)+\left(1-\epsilon_{\mathrm{t}}\right) C T_{\mathrm{s}}+H^{\mathrm{ex}}(p, T)$

where $\epsilon_{\mathrm{t}}$ is the total porosity and $H^{\mathrm{ex}}=m^{\mathrm{ex}} p_{1}$ represents the counterpart in Hounsfield units of the excess amount adsorbed per voxel volume, $m^{\text {ex }}$. As anticipated above, adsorption can be readily quantified by using as reference a tomogram acquired using an inert gas, such as helium $\left(H^{\mathrm{ex}}=0 \mathrm{HU}\right)$, at the reference $\left(p^{*}, T^{*}\right)$ conditions (Joss and Pini 2017):

$$
\begin{aligned}
& H^{\mathrm{ex}}(p, T)=\overline{C T}_{\mathrm{a}}(p, T)-\overline{C T}_{\mathrm{He}}\left(p^{*}, T^{*}\right) \\
& -\epsilon_{\mathrm{t}}\left(C T_{\mathrm{a}}(p, T)-C T_{\mathrm{He}}\left(p^{*}, T^{*}\right)\right)
\end{aligned}
$$

where $C T_{\mathrm{a}}(p, T)$ and $C T_{\mathrm{He}}\left(p^{*}, T^{*}\right)$ are the $\mathrm{CT}$ numbers of the adsorptive and of helium at the given pressure and temperature. Eq. 3 is the main operating equation to quantify adsorption by X-ray CT and can be applied to each voxel in the three-dimensional object to map adsorption spatially. When both the experiment and the reference scans are acquired at atmospheric conditions, $C T_{\mathrm{a}}(p, T) \approx C T_{\mathrm{He}}\left(p^{*}, T^{*}\right)$, and the third term in Eq. 3 can be safely neglected (Pini 2014; Joss and Pini 2017).

The ability of X-ray CT to measure adsorption depends on the strength of the change in the (bulk) density of the object upon exposure to the adsorptive gas. The question thus arises as to whether such change is strong enough for systems that are relevant for practical applications. Without loss of generality, we consider an adsorption column 
(bed porosity, $\epsilon=0.4$ ) packed with adsorbent pellets (density, $\rho_{\mathrm{p}}=900 \mathrm{~kg} / \mathrm{m}^{3}$ and specific micropore volume, $v_{\mathrm{m}}=1 \times 10^{-4} \mathrm{~m}^{3} / \mathrm{kg}$ ). Upon exposure to the adsorptive gas at ambient conditions, the change in the measured attenuation, $\Delta \overline{C T}$, can be estimated as follows:

$\Delta \overline{C T}=p_{1} \Delta \bar{\rho}=p_{1} \rho_{\mathrm{L}} v_{\mathrm{m}} \rho_{\mathrm{p}}(1-\epsilon)$

where $\rho_{\mathrm{L}}$ is the density of the adsorbed phase, which we assume to be equal to the density of the adsorptive at the boiling point at ambient pressure. For $\mathrm{CO}_{2}\left(\rho_{\mathrm{L}}=1278 \mathrm{~kg} / \mathrm{m}^{3}\right)$, $\mathrm{CH}_{4}\left(\rho_{\mathrm{L}}=422 \mathrm{~kg} / \mathrm{m}^{3}\right)$ and $\mathrm{N}_{2}\left(\rho_{\mathrm{L}}=809 \mathrm{~kg} / \mathrm{m}^{3}\right)$, the complete filling of the micropores with the adsorbed phase would generate a change in $\Delta \overline{C T}$ of about $70 \mathrm{HU}, 23 \mathrm{HU}$ and 44HU, respectively. A medical X-ray CT scanner can measure attenuation differences as low as $0.1 \%$ with crosssectional resolution of $0.01-0.05 \mathrm{~mm}^{2}$. However, for quantitative analysis image downscaling by super-sampling needs to be applied to yield voxel elements with a volume of about $10 \mathrm{~mm}^{3}$ and for which the uncertainty in the measured signal is about 3HU (Joss and Pini 2017), hence 4-10\% of the intensity estimated above. For larger volumes $\left(\sim 100 \mathrm{~mm}^{3}\right)$, such as when slice- and column-averaged properties are computed, this uncertainty becomes negligible $(<1 \%)$ (Joss and Pini 2017).

With the intent of investigating the adsorption properties of industrially relevant guest-host systems (e.g., $\mathrm{CO}_{2}$ on activated carbon or zeolite Pini 2014; Joss and Pini 2017, 2019), the experimental approach just presented exploits solely the attenuation contrast provided by the liquid-like adsorbed phase to map adsorption spatially. This is the approach also applied in this study. As an alternative to "conventional" process gases, strongly attenuating compounds can also be used, including krypton and xenon (Lu et al. 1994; Watson and Mudra 1994; Karacan and Okandan 2001), as well as brominated (Dubinin et al. 1975) or chlorinated (Lottaz and Lavanchy 1987; Lodewyckx et al. 2006) solvent vapours. By increasing the photoelectric contribution to the linear attenuation coefficient, these radio-opaque compounds provide a five- to hundred-fold increase in the measured attenuation, which can be exploited to study more weakly sorbing materials (Lu et al. 1994; Watson and Mudra 1994; Karacan and Okandan 2001; Vega et al. 2014) or to increase the spatial resolution of the characterisation (Mayo et al. 2015, 2018).

\section{Materials and methods}

Activated carbon (AC) rods (Norit RB3, nominal diameter $3 \mathrm{~mm}$, supplied by Sigma-Aldrich) were used as purchased. Prior to the experiments, the adsorbent material was regenerated at $423.15 \mathrm{~K}$ overnight. Basic textural characterisation of the adsorbent was carried out by physisorption analysis using Ar at $87 \mathrm{~K}$ in a Quantachrome Autosorb iQ to yield the following properties (Ansari et al. 2020): specific surface area, $1790 \mathrm{~m}^{2} / \mathrm{g}$; micro- $\left(0.46 \mathrm{~cm}^{3} / \mathrm{g}\right)$, meso- $\left(0.17 \mathrm{~cm}^{3} / \mathrm{g}\right)$ and macro-pore volume $\left(0.15 \mathrm{~cm}^{3} / \mathrm{g}\right)$. Assuming a solid (skeleton) density, $\rho_{\mathrm{sk}}=2.0 \mathrm{~g} / \mathrm{cm}^{3}$, the particle density is thus estimated to be $\rho_{\mathrm{p}}=0.78 \mathrm{~g} / \mathrm{cm}^{3}$, corresponding to a particle voidage, $\epsilon_{\mathrm{p}}=0.61$. The gases used in this study were procured from BOC, namely $\mathrm{Ar}$ and $\mathrm{He}$ at purities of $99.999 \%$ and $\mathrm{CO}_{2}$ at a purity of $99.995 \%$.

\subsection{Volumetry}

$\mathrm{CO}_{2}$ adsorption equilibrium isotherms have been measured in the pressure range $\mathrm{p}=0-101.325 \mathrm{kPa}$ at 293.15, 303.15, and $313.15 \mathrm{~K}$ using a Quantachrome Autosorb iQ. The sample $(1.7 \mathrm{~g})$ was activated for $12 \mathrm{~h}$ under $423.15 \mathrm{~K}$ prior to the isotherm measurements. A dual-site Langmuir adsorption isotherm model was applied to describe the experimental data:

$q^{*}=\frac{q_{\mathrm{s} 1} b_{1} c}{1+b_{1} c}+\frac{q_{\mathrm{s} 2} b_{2} c}{1+b_{2} c}$

where $q^{*}$ is the equilibrium amount adsorbed per unit volume and $c$ is the gas phase concentration; $q_{\mathrm{s} 1}$ and $q_{\mathrm{s} 2}$ are the saturation capacity values of the first and second site, respectively. The associated adsorption equilibrium constants, $b_{1}$ and $b_{2}$, depend on the temperature and abide the van't Hoff relationship:

$b_{i}=b_{0 i} e^{\frac{-\Delta U_{i}}{R T}} \quad$ for $i=1,2$

where $\Delta U_{i}$ is the change in internal energy due to adsorption for the two adsorption sites. The set of parameters $\left[q_{\mathrm{si}}, b_{0 i}, \Delta U_{i}\right]$ for $i=1,2$ was fitted to the experimental isotherms using the nonlinear regression function $\mathrm{nl}$ infit in MATLAB. The obtained parameter values are summarised in Table 1.

\subsection{Dynamic column breakthrough experiments}

The breakthrough experiments were conducted using the apparatus depicted in Fig. 1 and built in-house. It includes a $2.54 \mathrm{~cm}$-diameter, $28.6 \mathrm{~cm}$-long aluminium column packed

Table 1 Fitted dual-site Langmuir adsorption parameters for $\mathrm{CO}_{2}$ on activated carbon

\begin{tabular}{ll}
\hline Parameter & Value \\
\hline$b_{01}\left[\mathrm{~m}^{3} / \mathrm{mol}\right]$ & $3.17 \times 10^{-6}$ \\
$b_{02}\left[\mathrm{~m}^{3} / \mathrm{mol}\right]$ & $3.21 \times 10^{-6}$ \\
$q_{\mathrm{s} 1}[\mathrm{~mol} / \mathrm{kg}]$ & 0.44 \\
$q_{\mathrm{s} 2}[\mathrm{~mol} / \mathrm{kg}]$ & 6.10 \\
$-\Delta U_{1}[\mathrm{~kJ} / \mathrm{mol}]$ & 28.63 \\
$-\Delta U_{2}[\mathrm{~kJ} / \mathrm{mol}]$ & 20.37
\end{tabular}




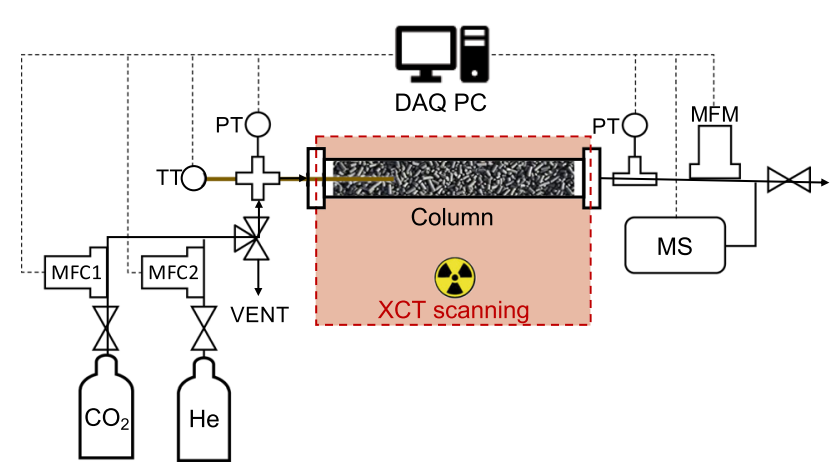

Fig. 1 Schematic of the dynamic column breakthrough apparatus. The aluminium column packed with the adsorbent is placed horizontally on the bed of the X-ray CT scanner to enable imaging of the adsorption/desorption fronts during the experiment. Inlet flow rates are controlled by two mass flow controllers (MFC1 and MFC2) and the outlet flow rate is measured by a mass flow meter (MFM). The temperature within the column is recorded with a K-type thermocouple (TT) inserted axially at a quarter of the column length. Pressure is recorded upstream and downstream of the column by means of two pressure transducers (PT). The outlet composition is measured and recorded with a mass spectrometer (MS)

with $34.7 \mathrm{~g}$ of AC rods that were regenerated overnight at 423.15 K prior to loading them into the column. Packing was performed under a gentle flow of helium to minimise contact with impurities present in air. Stainless steel wool was placed on both sides of the AC bed to allow for a good flow distribution. A thermocouple (1.6 $\mathrm{mm}$ sheath diameter, K-type) was positioned axially at a quarter of the column length $(72 \mathrm{~mm})$ to monitor internal temperature changes, and a second exposed thermocouple was fixed on the outer side of the aluminium column at the same axial position. Two mass flow controllers (MFC1, model MC-200SCCM-D and MFC2, model MC-500SCCM-D, by Alicat, USA) and a mass flow meter (MFM, model M-1SLPM-D, by Alicat, USA) were used to control the inlet $\mathrm{He} / \mathrm{CO}_{2}$ flow rates and to measure the outlet flow rate, respectively.

The two MFCs and the MFM were calibrated prior to the experimental campaign with a volumetric flow analyzer (ADM Flow Meter by Agilent technologies, USA); pure gas calibration curves were measured for the MFCs, and a bivariate calibration curve as a function of flow rate and gas composition was measured for the MFM. Pressure changes and pressure drop during the experiments were recorded with two pressure transducers (model PA$33 \mathrm{X}, 0$ bar to 40 bar, Keller, Switzerland) placed at the inlet and outlet of the column, respectively. The dynamic breakthrough measurements were monitored by measuring the outlet gas phase composition with a mass spectrometer (MS, model GSD 320 O1, OmniStar Gas Analysis system by Pfeiffer Vacuum, Switzerland). Prior to the first experiment, the packed column was regenerated overnight
Table 2 Summary of the experimental conditions of the dynamic adsorption/desorption experiments

\begin{tabular}{llll}
\hline Mode & $y_{\text {in }}$ & $f_{\text {in }}$ & \# scans \\
\hline Ads & 1 & 50 & 36 \\
Des & 0 & 100 & 20 \\
Ads & 1 & 100 & 25 \\
Des & 0 & 150 & 16 \\
Ads & 1 & 200 & 36 \\
Des & 0 & 250 & 16 \\
Ads & 0.5 & 100 & 29 \\
\hline
\end{tabular}

All experiments were carried out under ambient temperature $(293.15 \mathrm{~K})$ and pressure $(100 \mathrm{kPa})$. The injection flow rate, $f_{\text {in }}$, is given in $\mathrm{cm}^{3} / \mathrm{min}$

by flowing $\mathrm{He}\left(25 \mathrm{~cm}^{3} / \mathrm{min}\right)$ at ambient pressure and temperature, followed by the acquisition of a X-ray CT scan of the whole column. A total of three $\mathrm{CO}_{2}$ dynamic adsorption/desorption experiments were carried out at ambient temperature $(293.15 \mathrm{~K})$ and pressure $(100 \mathrm{kPa})$, while varying flow rates, $f_{\text {in }}$, as listed in Table 2 . Additionally, one dynamic adsorption experiment was carried out using a 50:50 $\mathrm{CO}_{2}: \mathrm{He}$ mixture $\left(f_{\text {in }}=100 \mathrm{~cm}^{3} / \mathrm{min}\right)$ at ambient temperature and pressure. For each dynamic adsorption experiment, the column was first purged with helium; at time $t=0$, the experiment was started by flowing either pure $\mathrm{CO}_{2}$ or $\mathrm{CO}_{2} / \mathrm{He}$ through the column and X-ray CT scans of the whole column were acquired at regular time intervals (Sect. 3.4). The adsorption experiment was completed once the concentration and temperature of the outlet gas was equal to that of the feed. At this point, a desorption experiment was initiated by flowing helium through the column and again X-ray CT scans of the whole column were acquired. The experiment was stopped when the detected $\mathrm{CO}_{2}$ concentration was less than $100 \mathrm{ppm}$ and the temperature in the column was equal to that of the feed.

A series of static adsorption experiments were conducted at the end of the experimental campaign. To this end, the column was saturated with $\mathrm{CO}_{2}: \mathrm{He}$ mixtures of varying composition $(\{25: 75 ; 50: 50 ; 75: 25 ; 100: 0\})$ at a total pressure of $100 \mathrm{kPa}$ and $293.15 \mathrm{~K}$. At each composition step, a $\mathrm{X}$-ray CT scan of the whole adsorption column was acquired and used to calculate the average adsorbed amount over the column section that did not contain the thermocouple (slices between $90 \mathrm{~mm}$ and $240 \mathrm{~mm}$ from the inlet).

\subsection{Mass balance calculations}

The amount of gas adsorbed for any specific breakthrough run can be calculated from the $\mathrm{CO}_{2}$ mass balance around the adsorption column: 
$\int_{0}^{t}\left(\frac{f_{\text {in }} p_{\text {in }} y_{\text {in }}}{R T_{\text {in }}}\right) d t-\int_{0}^{t}\left(\frac{f_{\text {out }} p_{\text {out }} y_{\text {out }}}{R T_{\text {out }}}\right) d t=\rho_{\mathrm{b}} V_{\mathrm{b}} q_{\mathrm{bt}}^{*}+\frac{\epsilon V_{\mathrm{b}} \bar{p} y_{\text {in }}}{R T}$

where the first and the second terms on the LHS of Eq. 7 are the amount of $\mathrm{CO}_{2}$ entering and leaving the column for the duration of experiment, respectively, while the first and second term on the RHS of the equation represent the amounts of $\mathrm{CO}_{2}$ adsorbed and accumulated in the bulk gas phase, respectively. $f, p, y$, and $T$ are the recorded volumetric flow rate, pressure, $\mathrm{CO}_{2}$ mole fraction, and temperature, respectively ( $\bar{p}$ is the arithmetic mean of inlet and outlet pressures). $V_{\mathrm{b}}$ is the bed volume, $\epsilon$ is the bed porosity and $\rho_{\mathrm{b}}$ is the bed density. Therefore, one can rearrange Eq. 7 to calculate the amount of $\mathrm{CO}_{2}$ adsorbed, $q_{\mathrm{bt}}^{*}$, which can be compared to independent equilibrium measurements by (i) volumetry $\left(q^{*}\right.$, Sect. 3.1) and (ii) X-ray CT ( $q_{\mathrm{ct}}^{*}$, Sect. 3.4).

\subsection{XCT imaging}

X-ray CT scans of the whole column were taken prior to each adsorption experiment, when the adsorbent was exposed to helium, as well as during each dynamic adsorption/desorption experiment. Scanning was carried out in a Toshiba Aquilon 64 scanner in helical mode by applying a peak tube voltage of $120 \mathrm{kV}$ and a current of $200 \mathrm{~mA}$. The images were reconstructed with a $x y z$ resolution of $0.07 \times 0.07 \times 1 \mathrm{~mm}$ without applying any filter. Each $3 \mathrm{D}$ scan took ca. $10 \mathrm{~s}$ to complete; the total number of scans taken during each dynamic experiment is listed in Table 2. Image analysis was carried out on a $14 \mathrm{~mm}$-diameter, $240 \mathrm{~mm}$-long cylindrical segment of the packed bed, as indicated in Fig. 2a, b. This cropping of the images was implemented to avoid any imaging artefacts arising at the column wall and at the inlet and outlet of the column (which where fitted with stainless steel compression fittings). Raw images based on CT numbers are shown in Fig. 2c,e for two selected slices at three different times during an adsorption and a desorption experiment. Changes in the local attenuation due to adsorption become readily apparent when difference images are considered, as shown in panels $\mathrm{d}$ and $\mathrm{f}$ for the $3 \mathrm{D}$ column at the same times. These difference images are used to compute the amount adsorbed per unit mass of adsorbent as a function of position and time, $q_{\mathrm{ct}}(\mathbf{x}, t)$. Specifically, the attenuation measured at a given time $t$ and position $\mathbf{x}$ upon exposure to the adsorptive, $\overline{C T}_{\mathrm{a}}(\mathbf{x}, t)$, is combined with the corresponding value recorded in the registered scan of the bed saturated with helium, $\overline{C T}_{\mathrm{He}}(\mathbf{x})=\overline{C T}_{\mathrm{a}}(\mathbf{x}, t=0)$ :

$q_{\mathrm{ct}}(\mathbf{x}, t)=\frac{\overline{C T}_{\mathrm{a}}(\mathbf{x}, t)-\overline{C T}_{\mathrm{He}}(\mathbf{x})}{p_{1} M_{\mathrm{m}} \rho_{\mathrm{b}}}$

where $M_{\mathrm{m}}=0.044 \mathrm{~kg} / \mathrm{mol}$ is the molar mass of $\mathrm{CO}_{2}, \rho_{\mathrm{b}}$ is the bed density and $p_{1}=1 \mathrm{HU} \mathrm{m}^{3} / \mathrm{kg}$. Eq. 8 was applied to compute pellet-, slice- or column-averaged amount adsorbed by using pellet-, slice- or column-averaged CT numbers, respectively. As described in Sect. S1, the uncertainty associated with pellet-averaged adsorption values $\left(V \approx 20 \mathrm{~mm}^{3}\right)$ is about $q_{\mathrm{ct}} \pm 100 \mu \mathrm{mol} / \mathrm{g}\left(\rho_{\mathrm{p}} \approx 0.8 \mathrm{~g} / \mathrm{cm}^{3}\right)$, while it amounts to $q_{\mathrm{ct}} \pm 30 \mu \mathrm{mol} / \mathrm{g}$ and below for slice- or columnaveraged CT numbers $\left(V>200 \mathrm{~mm}^{3}, \rho_{\mathrm{b}} \approx 0.4 \mathrm{~g} / \mathrm{cm}^{3}\right)$.

To extract adsorption isotherms of individual pellets within the adsorbent column, the X-ray CT images were processed using the marker-controlled watershed segmentation algorithm implemented in MATLAB and described in Sect. S2. The algorithm was applied to a X-ray CT scan of the column saturated with helium and the obtained mask was then used to extract pellet-by-pellet adsorption data from the scans acquired during $\mathrm{CO}_{2}$ adsorption. In this study, this detailed analysis was limited to a $1 \mathrm{~cm}$-thick section of the column located at a distance of $7.4-8.4 \mathrm{~cm}$ from the inlet to yield transient and equilibrium adsorption data for 12 individual pellets.

\section{Modelling adsorption dynamics}

We present in this section the modeling approach that has been used to describe the experimental breakthrough curves as well as the internal profiles, together with the procedure adopted to estimate all relevant model parameters.

\subsection{Equilibrium considerations}

When applied to gas separations, the analysis of wave phenomena by means of the equilibrium theory provides a useful method to predict the propagation of composition fronts within the adsorption column (Mazzotti and Rajendran 2013). With relation to the experiments discussed in this work, the injection of a feed stream rich in $\mathrm{CO}_{2}\left(y_{\text {in }}>0\right)$ into the column initially regenerated $(y=0)$ yields the formation of a shock front that propagates through the column at the velocity, $v_{\text {sh }}$ (Kayser and Knaebel 1989):

$v_{\mathrm{sh}}=\frac{v_{2}}{1+\frac{\rho_{\mathrm{b}}}{\epsilon}\left(\frac{q^{*}\left(c_{\text {in }}\right)}{y_{\text {in }}}\right) \frac{R T}{\bar{p}}}$

where $v_{2}$ is the interstitial gas velocity at the trailing edge of the shock front $\left(f_{\text {in }}=\epsilon A v_{2}\right)$. The latter is related to the gas velocity at the leading edge of the shock front, $v_{1}$, by the following relationship (Kayser and Knaebel 1989):

$v_{1}=v_{2}+y_{\mathrm{F}}\left(v_{\mathrm{sh}}-v_{2}\right)$

Therefore, given the feed composition and the corresponding equilibrium amount adsorbed, one can readily predict the breakthrough time of the shock front at the column outlet, 
a

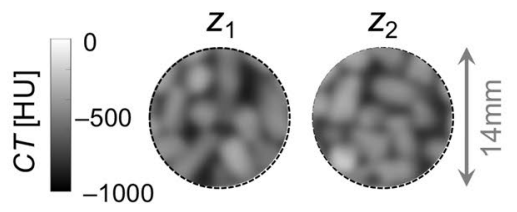

C

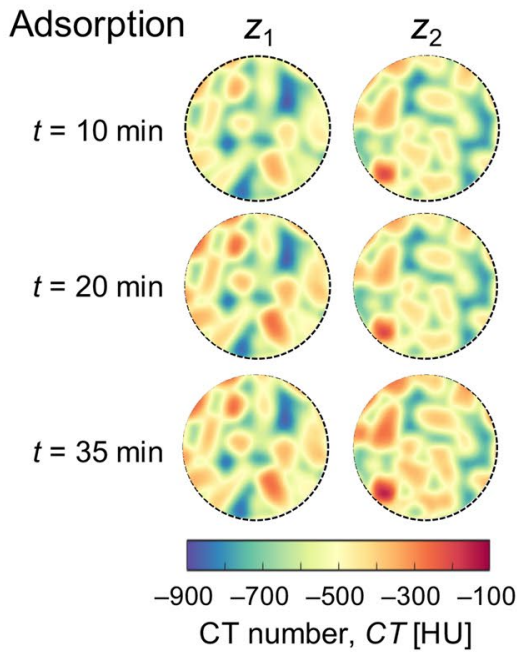

e

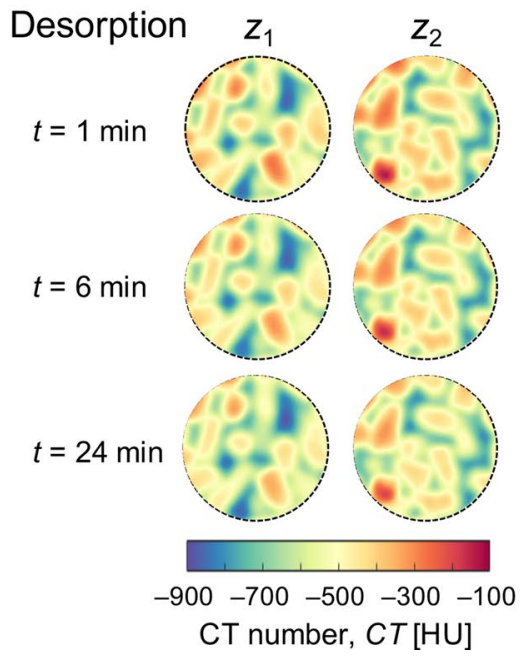

Fig. 2 Monitoring gas adsorption dynamics in space and time. A $14 \mathrm{~mm}$-diameter, $240 \mathrm{~mm}$-long cylinder is cropped from the raw 3D scan for image analysis $(\mathbf{a}, \mathbf{b})$. Two selected slices at positions $z_{1}, z_{2}$ (80 and $160 \mathrm{~mm}$ from the inlet) are shown in a, c, e to illustrate the image resolution $(0.07 \times 0.07 \times 1 \mathrm{~mm})$ and the variation in X-ray attenuation in Hounsfield units. The amount adsorbed

$t_{\mathrm{bt}}^{*}=L / v_{\mathrm{sh}}$, or at any given internal location $z, t_{\mathrm{bt}}^{*}(z)=z / v_{\mathrm{sh}}$, as well as the flow rate observed at the outlet of the column prior to breakthrough $\left(f_{\text {out }}^{*}=\epsilon A v_{1}\right)$. b

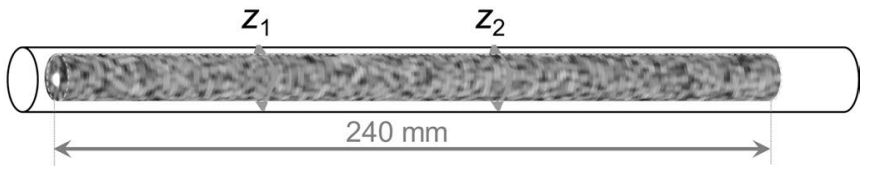

d

$\mathrm{CO}_{2}$
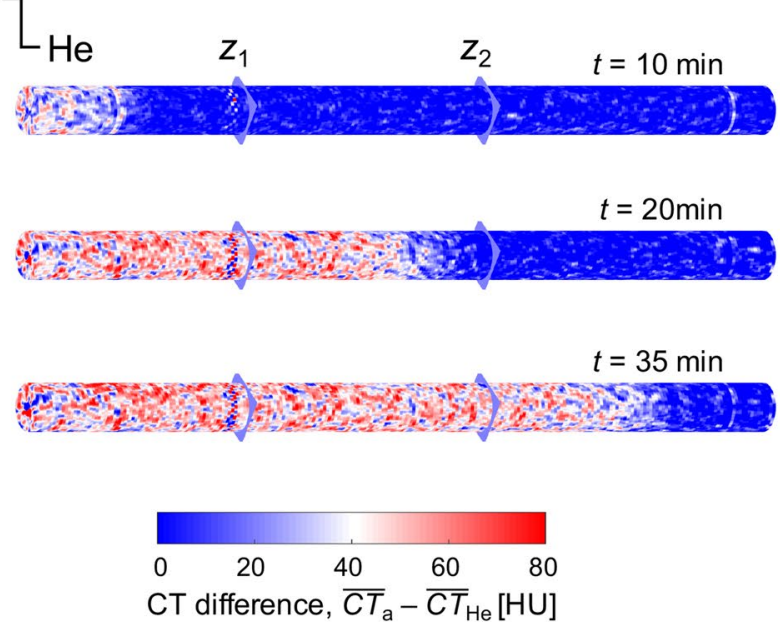

f

$\mathrm{He}$

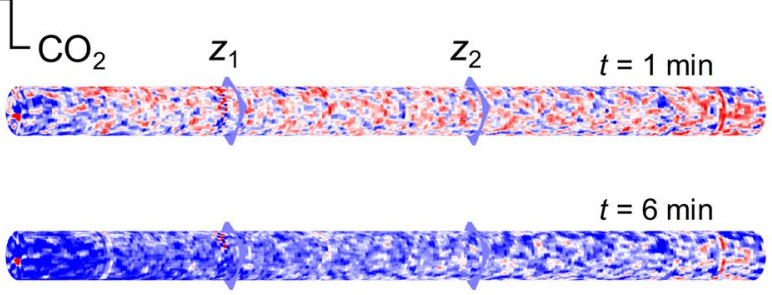

$t=24 \min$

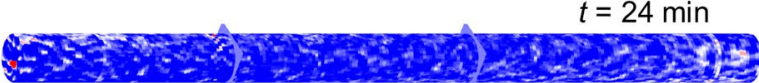

is computed upon subtracting a registered helium X-ray CT scan from the one obtained during the dynamic experiment. These difference scans are shown for three different times for $\mathbf{d}$ an adsorption experiment at $f_{\text {in }}=50 \mathrm{~cm}^{3} / \mathrm{min}$ and $\mathbf{f}$ a desorption experiment at $f_{\text {in }}=100 \mathrm{~cm}^{3} / \mathrm{min}$

\subsection{Dynamic column breakthrough simulation}

A generic one-dimensional model was used to simulate the dynamic adsorption/desorption experiments. The model is 
described in detail in Hosseinzadeh Hejazi et al. (2016) and includes contributions from hydrodynamic dispersion, convection, adsorption, heat transfer, and pressure drop across the column. Briefly, the axially-dispersed plug flow model is used to describe the flow of an ideal gas phase through the adsorption column. Darcy's law is used to compute the pressure drop along the column. Mass transfer in the solid phase is formulated using a linear driving force (LDF) model with macropore diffusion control. Convective and conductive heat transfer mechanisms are described to occur inside and outside the column (radiant heat is neglected), and gas and solid phases are assumed to be at thermal equilibrium. Experimental mole fraction, temperature, and flow rate profiles are simulated by solving the following mass and energy balances: overall and component mass balances for the fluid phase, component mass balances for the solid phase, and energy balances both inside the column and on its wall. The associated partial differential equations are listed in Table 3. These equations have been discretized in space with the finite volume technique (Haghpanah et al. 2013) and integrated using MATLAB's ode15s solver. In this study, the number of components $n_{\mathrm{c}}=2$ with component 2 being the inert carrier gas (helium, $q^{*}=0$ ).

\subsection{Parameter estimation}

The physical properties of the adsorbent, the fluid phase and the column are introduced as an input to the model and are listed in Table 4. The internal and external heat transfer coefficients were determined by optimization to match the composition and thermal breakthrough profiles of the adsorption experiments at each experimental flow rate independently. The reason for this is that precise temperature control around the column could not be achieved, as it was not practical to use a thermal bath due to the need for simultaneous imaging. Additionally, the dissipated heat from X-ray source may also impact the internal temperature profiles during the scanning time and this effect can vary for each flow rate depending on the frequency of the scans. The values of heat transfer coefficients fitted to the adsorption experiment with the lowest flow rate were used in a predictive manner to simulate experimental desorption profiles. To estimate the values of the adjustable parameters, the non-dominated sorting genetic algorithm II (GA) in the MATLAB global optimization toolbox was used with the following objective function to be minimized:

$$
\begin{aligned}
& J=\frac{1}{1000 N_{\mathrm{y}}} \sum_{j=1}^{N_{\mathrm{y}}}\left(\frac{y_{\text {out }}^{\exp }\left(t_{j}\right)-y_{\text {out }}^{\mathrm{sim}}\left(t_{j}\right)}{y_{\text {out }}^{\exp }\left(t_{j}\right)}\right)^{2}+\frac{1}{N_{\mathrm{T}}} \sum_{j=1}^{N_{\mathrm{T}}} \\
& \left(\frac{T_{\mathrm{tc}}^{\exp }\left(t_{j}\right)-T^{\operatorname{sim}}\left(t_{j}\right)}{T_{\mathrm{tc}}^{\exp }\left(t_{j}\right)}\right)^{2}
\end{aligned}
$$

where $N_{\mathrm{y}}$ and $N_{\mathrm{T}}$ are the number of time steps, $t_{j}$, in the recorded composition and temperature profiles, $y_{\text {out }}^{\text {xxp }}$ is the outlet mole fraction measured by the mass spectrometer and $y_{\text {out }}^{\text {sim }}$ is its value predicted by the model. $T^{\mathrm{sim}}$ and $T_{\mathrm{tc}}^{\exp }$ are the temperatures values of the gas phase at a distance of $7.2 \mathrm{~cm}$ from the inlet predicted by the model and measured by the thermocouple located inside the column, respectively. We note that including the residuals of both the mole fraction and temperature in the objective function ensures that both profiles are correctly captured by the model.

\begin{tabular}{|c|c|}
\hline Overall mass balance & $\frac{1}{P} \frac{\partial P}{\partial t}-\frac{1}{T} \frac{\partial T}{\partial t}=-\frac{T}{P} \frac{\partial}{\partial z}\left(\frac{P}{T} v\right)-\frac{R T}{P} \frac{\rho_{\mathrm{b}}}{\epsilon} \sum_{i=1}^{n_{\mathrm{c}}} \frac{\partial q_{i}}{\partial t}$ \\
\hline Component mass balance & $\frac{\partial y_{i}}{\partial t}+\frac{y_{i}}{P} \frac{\partial P}{\partial t}-\frac{y_{i}}{T} \frac{\partial T}{\partial t}=\frac{T}{P} D_{L} \frac{\partial}{\partial z}\left(\frac{P}{T} \frac{\partial y_{i}}{\partial z}\right)^{i=1}-\frac{T}{P} \frac{\partial}{\partial z}\left(\frac{y_{i} P}{T} v\right)-\frac{R T}{P} \frac{\rho_{\mathrm{b}}}{\epsilon} \frac{\partial q_{i}}{\partial t}$ \\
\hline Solid phase mass balance & $\frac{\partial q_{i}}{\partial t}=k_{i}\left(q_{i}^{*}-q_{i}\right), k_{i}=\frac{15 \epsilon_{\mathrm{p}} D_{\mathrm{m}}}{\tau r_{\mathrm{p}}^{2}}$ \\
\hline Pressure drop & $-\frac{\partial P}{\partial z}=\frac{150}{4} \frac{1}{r_{\mathrm{p}}^{2}}\left(\frac{1-\epsilon}{\epsilon}\right)^{2} \mu \nu$ \\
\hline Column energy balance & $\begin{array}{l}{\left[\frac{\rho_{\mathrm{b}}}{\epsilon}\left(C_{\mathrm{p}, \mathrm{s}}+C_{\mathrm{p}, \mathrm{a}} \sum_{i=1}^{n_{c}} q_{i}\right)\right] \frac{\partial T}{\partial t}=\frac{K_{\mathrm{z}}}{\epsilon} \frac{\partial^{2} T}{\partial^{2} z}-\frac{C_{\mathrm{p}, \mathrm{g}}}{R} \frac{\partial}{\partial z}(v P)-\frac{C_{\mathrm{p}, \mathrm{g}}}{R} \frac{\partial P}{\partial t}-\frac{\rho_{\mathrm{b}}}{\epsilon} C_{\mathrm{p}, \mathrm{a}} T \sum_{i=1}^{n_{\mathrm{c}}} \frac{\partial q_{i}}{\partial t}} \\
+\frac{\rho_{\mathrm{b}}}{\epsilon} \sum_{i=1}^{n_{\mathrm{c}}}\left(\left(-\Delta U_{i}+R T\right) \frac{\partial q_{i}}{\partial t}\right)-\frac{2 h_{\mathrm{in}}}{\epsilon r_{\mathrm{in}}}\left(T-T_{\mathrm{w}}\right)\end{array}$ \\
\hline Wall energy balance & $\rho_{\mathrm{w}} C_{\mathrm{p}, \mathrm{w}} \frac{\partial T_{\mathrm{w}}}{\partial t}=K_{\mathrm{w}} \frac{\partial^{2} T_{\mathrm{w}}}{\partial^{2} z}+\frac{2 r_{\text {in }} h_{\text {in }}}{r^{2}-r^{2}}\left(T-T_{\mathrm{w}}\right)-\frac{2 r_{\text {out }} h_{\text {out }}}{r^{2}-r^{2}}\left(T_{\mathrm{w}}-T_{\mathrm{a}}\right)$ \\
\hline
\end{tabular}

Table 3 Equations to simulate the dynamic adsorption/desorption experiments

Wall energy balance

$\rho_{\mathrm{w}} C_{\mathrm{p}, \mathrm{w}} \frac{\partial T_{\mathrm{w}}}{\partial t}=K_{\mathrm{w}} \frac{\partial^{2} T_{\mathrm{w}}}{\partial^{2} z}+\frac{2 r_{\text {in }} h_{\text {in }}}{r_{\text {out }}^{2}-r_{\text {in }}^{2}}\left(T-T_{\mathrm{w}}\right)-\frac{2 r_{\text {out }} h_{\text {out }}}{r_{\text {out }}^{2}-r_{\text {in }}^{2}}\left(T_{\mathrm{w}}-T_{\mathrm{a}}\right)$ 
Table 4 Parameters used to simulate the dynamic adsorption/desorption experiments

\begin{tabular}{|c|c|c|}
\hline Parameter & Value & Source \\
\hline \multicolumn{3}{|l|}{ Column dimensions and properties } \\
\hline Column length, $L[\mathrm{~m}]$ & 0.286 & Measured \\
\hline Inner column radius, $r_{\text {in }}[\mathrm{m}]$ & 0.00945 & Measured \\
\hline Outer column radius, $r_{\text {out }}[\mathrm{m}]$ & 0.0127 & Measured \\
\hline Column void fraction, $\epsilon$ & 0.456 & Measured \\
\hline Bed density, $\rho_{\mathrm{b}}\left[\mathrm{kg} \mathrm{m}^{-3}\right]$ & 435 & Measured \\
\hline \multicolumn{3}{|l|}{ Properties and constants } \\
\hline Particle voidage, $\epsilon_{p}$ & 0.59 & Assumed \\
\hline Tortuosity, $\tau$ & 3 & Assumed \\
\hline Universal gas constant, $R\left[\mathrm{~m}^{3} \mathrm{~Pa} \mathrm{~mol}^{-1} \mathrm{~K}^{-1}\right]$ & 8.314 & Standard value \\
\hline Column wall density, $\rho_{\mathrm{w}}\left[\mathrm{kg} \mathrm{m}^{-3}\right]$ & 2700 & Standard value for aluminum \\
\hline Specific heat capacity, $C_{\mathrm{p}, \mathrm{g}}\left[\mathrm{J} \mathrm{kg}^{-1} \mathrm{~K}^{-1}\right]$ & $840\left(\mathrm{CO}_{2}\right)$ & Standard values \\
\hline Specific heat capacity of adsorbed phase, $C_{\mathrm{p}, \mathrm{a}}\left[\mathrm{J} \mathrm{kg}^{-1} \mathrm{~K}^{-1}\right]$ & $840\left(\mathrm{CO}_{2}\right)$ & Assumed \\
\hline Specific heat capacity of adsorbent, $C_{\mathrm{p}, \mathrm{s}}\left[\mathrm{J} \mathrm{kg}^{-1} \mathrm{~K}^{-1}\right]$ & 1040 & Rufford et al. (2013) \\
\hline Specific heat capacity of column wall, $C_{\mathrm{p}, \mathrm{w}}\left[\mathrm{J} \mathrm{kg}^{-1} \mathrm{~K}^{-1}\right]$ & 902 & Standard value for aluminum \\
\hline Fluid viscosity, $\mu\left[\mathrm{kg} \mathrm{m}^{-1} \mathrm{~s}^{-1}\right]$ & $1.720 \times 10^{-5}\left(\mathrm{CO}_{2}\right)$ & Standard values \\
\hline Molecular diffusivity, $D_{\mathrm{m}}\left[\mathrm{m}^{2} \mathrm{~s}^{-1}\right]$ & $0.605 \times 10^{-5}\left(\mathrm{CO}_{2}\right)$ & Standard values \\
\hline Effective gas thermal conductivity, $K_{\mathrm{z}}\left[\mathrm{J} \mathrm{m}^{-1} \mathrm{~K}^{-1} \mathrm{~s}^{-1}\right]$ & 1 & Assumed \\
\hline Thermal conductivity of column wall, $K_{\mathrm{w}}\left[\mathrm{J} \mathrm{m}^{-1} \mathrm{~K}^{-1} \mathrm{~s}^{-1}\right]$ & 205 & Standard value for aluminum \\
\hline Inside heat transfer coefficient, $h_{\mathrm{in}}\left[\mathrm{J} \mathrm{m}^{-2} \mathrm{~K}^{-1} \mathrm{~s}^{-1}\right]$ & $\begin{array}{l}61.50\left(50 \mathrm{~cm}^{3} / \mathrm{min}\right), 50.14\left(100 \mathrm{~cm}^{3} /\right. \\
\mathrm{min}), 35.23\left(200 \mathrm{~cm}^{3} / \mathrm{min}\right)\end{array}$ & Fitted for each flow rate \\
\hline Outside heat transfer coefficient, $h_{\text {out }}\left[\mathrm{J} \mathrm{m}^{-2} \mathrm{~K}^{-1} \mathrm{~s}^{-1}\right]$ & $\begin{array}{l}6.46\left(50 \mathrm{~cm}^{3} / \mathrm{min}\right), 7.12\left(100 \mathrm{~cm}^{3} /\right. \\
\mathrm{min}), 5.88\left(200 \mathrm{~cm}^{3} / \mathrm{min}\right)\end{array}$ & Fitted for each flow rate \\
\hline
\end{tabular}

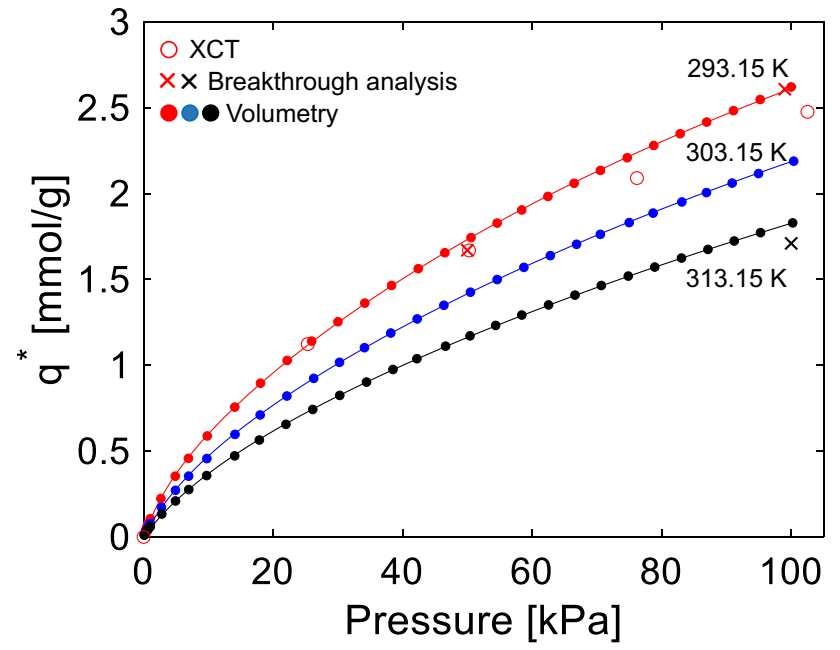

Fig. $3 \mathrm{CO}_{2}$ adsorption equilibrium isotherms on activated carbon rods. Solid circles are experimental values from volumetry and lines are fitted dual-site Langmuir model isotherms. The cross symbols are results from breakthrough experiments calculated from mass balance calculations. Empty circles are results from X-ray CT imaging experiments

\section{Results}

\section{$5.1 \mathrm{CO}_{2}$ adsorption isotherms}

As described in Sect. 3, $\mathrm{CO}_{2}$ adsorption isotherms were measured using three independent techniques, namely volumetry, breakthrough analysis (mass balance) and imaging by X-ray CT. These data are shown as symbols in Fig. 3, together with the fits obtained upon application of the dualsite Langmuir model (solid lines) to the volumetry data (solid circles). The latter have been obtained at three different temperatures $(293.15,303.15$, and $313.15 \mathrm{~K}$ ), so as to provide an accurate parameterization of heat effects in the description of the dynamic adsorption experiments through the isosteric heat of adsorption, $-\Delta U$. It can be seen that the dual-site Langmuir model describes the experimental data accurately across the entire pressure range and at each temperature. The isotherm model parameters are listed in Table 1.

Results obtained by breakthrough analysis (crosses) and by X-ray CT imaging (empty circles) are also shown in the figure and show good agreement with the independent volumetry data. We note that the cross symbol at $293.15 \mathrm{~K}$ and $100 \mathrm{kPa}$ represents an average of six unique experimental adsorption and desorption runs carried out at three different 
flow rates. The small variation among the six different runs $\left(q_{\mathrm{bt}}^{*} \pm 0.15 \mathrm{mmol} / \mathrm{g}\right)$ confirms the accuracy of our experimental protocol for breakthrough measurements. Similarly, the empty circles at $293.15 \mathrm{~K}$ and $25,50,75$, and $100 \mathrm{kPa}$ have been estimated from four independent X-ray CT scans at each pressure value and demonstrate excellent reproducibility of the imaging-based method $\left(q_{\mathrm{ct}}^{*} \pm 0.01 \mathrm{mmol} / \mathrm{g}\right)$. We note, however, that with increasing pressure the X-ray CT data deviate from the other two sets of measurements towards lower adsorption values, reaching an absolute difference of $-0.125 \mathrm{mmol} / \mathrm{g} \mathrm{(5 \% )}$ at about $100 \mathrm{kPa}$. Albeit small, we attribute this systemtatic error to the calibration factor $p_{1}$ in Eq. 8, which in this study was kept to the instrument's factory settings, rather than carrying out an ad-hoc calibration. Nevertheless, the general agreement observed in Fig. 3 among the equilibrium adsorption data provides a solid basis for the comparison between the the internal adsorption profiles and those predicted using the dynamic adsorption column model, as discussed in Sect. 5.3.

\subsection{Adsorption dynamics: conventional output}

Traditionally, the output of a dynamic breakthrough experiment includes transient data for the outlet gas composition and volumetric flow rate, as well as for the temperature measured at one or more locations within the adsorbent column. These data are shown as symbols in Fig. 4 for the experiments carried out at $293.15 \mathrm{~K}$ and at three different feed flow rates $\left(f_{\text {in }}=50,100\right.$, and $\left.200 \mathrm{~cm}^{3} / \mathrm{min}\right)$. In the figure, the three top panels refer to (a) outlet mole fraction, (b) internal temperature profile and (c) outlet flow rate obtained during each adsorption step. The results associated with the subsequent desorption step are presented in three bottom panels $\left(f_{\text {in }}=100,150\right.$, and $250 \mathrm{~cm}^{3} / \mathrm{min}$, same color-coding). The results obtained upon application of the dynamic adsorption column model are represented by the solid lines. As described in Sect. 4.3, only the observations from the adsorption steps were used to obtain estimates of the internal and external heat transfer coefficients by fitting (Table 4), while the model was applied here in a predictive manner to describe the observations from the desorption steps. The minimum value of the objective function (Eq. 11) was found to be $8.0 \times 10^{-4}, 5.5 \times 10^{-4}, 7.3 \times 10^{-4}$ for $f_{\text {in }}=50,100$, and $200 \mathrm{~cm}^{3} / \mathrm{min}$, respectively.

The model describes accurately the experimental outlet profiles of the gas composition (Fig. 4a) and flow rate (Fig. 4c) during each adsorption step. The sharp breakthrough observed verifies the fast mass transfer that can be
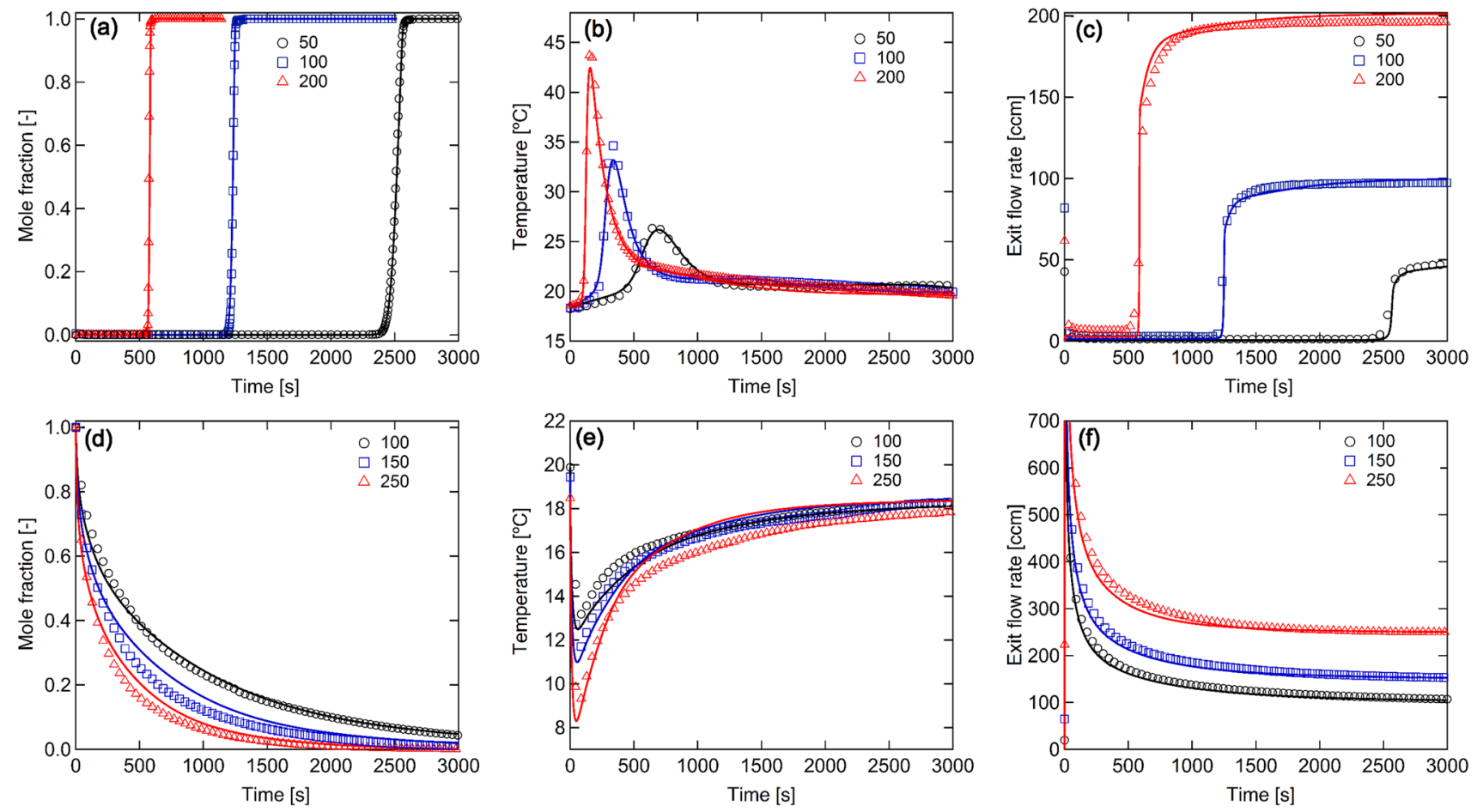

Fig. 4 Conventional output from $\mathrm{CO}_{2} / \mathrm{He}$ dynamic column breakthrough experiments at three different flow rates. The three top panels show the temporal evolution of the a outlet composition, $\mathbf{b}$ internal temperature $(7.2 \mathrm{~cm}$ from inlet) and $\mathbf{c}$ outlet flow rate observed during a $\mathrm{CO}_{2}$ feed $\left(y_{\text {in }}=1\right)$. Panels $\mathbf{d}$, e, $\mathbf{f}$ show the corresponding observa- tions during the subsequent helium purge ( $y_{\text {in }}=0$, same color coding, flow rates indicated in the legend). Symbols and lines correspond to experimental and simulation data, respectively. Experimental conditions: $p=100 \mathrm{kPa}, T=293.15 \mathrm{~K}$; the units of the flow rate are $\mathrm{cm}^{3} / \min$ 
achieved for $\mathrm{CO}_{2}$ on activated carbon rods. Accordingly, at breakthrough the outlet flow rate jumps from a value near zero to the corresponding feed flow rate, $f_{\text {in }}$. The behaviour is predicted reasonably well also by the equilibrium theory (Sect. 4.1). The outlet flow rate prior to breakthrough takes a finite value and shows a subtle-yet noticeableincrease with increasing value of $f_{\text {in }}\left(f_{\text {out }}^{*}=0.8,1.6\right.$ and $3.2 \mathrm{~cm}^{3} /$ min for $f_{\text {in }}^{*}=50,100$ and $200 \mathrm{~cm}^{3} / \mathrm{min}$ ). The predicted breakthrough times of the shock wave are, $t_{\mathrm{bt}}^{*}=2706 \mathrm{~s}\left(50 \mathrm{~cm}^{3} / \mathrm{min}\right), t_{\mathrm{bt}}^{*}=1353 \mathrm{~s}\left(100 \mathrm{~cm}^{3} / \mathrm{min}\right)$ and $t_{\mathrm{bt}}^{*}=677 \mathrm{~s}\left(200 \mathrm{~cm}^{3} / \mathrm{min}\right)$ and are $7.8 \%, 9.8 \%$, and $18.1 \%$ larger than the values observed experimentally. This overestimation is expected and is the result of adsorption heat effects; accordingly, larger deviations are observed at higher flow rates, when heat effects are more pronounced (see below). This is also visible in the outlet flow rate profile, which shows a slower transition to the feed value at higher flow rates.

The internal temperature profiles (Fig. 4b, e) evidence the significant temperature variations arising upon adsorption (temperature increase) and desorption (temperature decrease). These are well captured by the model, which also correctly represents the effect of the feed flow rate: with increasing value of $f_{\text {in }}$ the observed temperature peaks become larger. As opposed to the sharp transitions observed upon adsorption, both outlet composition (Fig. 4d) and flow rate (Fig. 4f) decrease gradually during the desorption steps. This is the expected behaviour for a type I adsorption isotherm: when the inlet concentration $\left(y_{\text {in }}\right)$ is smaller than the initial concentration $\left(y^{0}\right)$, the leading state $\left(y^{0}\right)$ travels faster than the trailing state $\left(y_{\text {in }}\right)$ and a so-called simplewave is formed (Mazzotti and Rajendran 2013). The latter is an expanding wave characterised by concentrations that monotonically decrease from $y^{0}=1$ to $y_{\text {in }}=0$. Because the velocity depends on the composition (Kayser and Knaebel 1989), its evolution over time (panel f) will mimic the evolution of the composition (panels d). Again, the model accurately describes these transient profiles.

\subsection{Adsorption dynamics: internal profiles}

X-ray CT imaging performed during the dynamic adsorption/ desorption experiments enables tracking the adsorption front as it progresses through the adsorbent column. The results obtained at different flow rates for the $\mathrm{CO}_{2}$ feed step $\left(y_{\text {in }}=1\right.$, left panels) and the subsequent helium purge ( $y_{\text {in }}=0$, right panels) are shown in Fig. 5. The color scale and labels indicate the time of each profile with respect to the residence time $t_{r}$ calculated as the volume of the empty column divided by the flow rate. The characteristic pattern of the internal profiles of the adsorbed concentration observed by X-ray CT during adsorption and desorption is consistent with the observations from the outlet composition profiles discussed above. The shape and velocity of the mass transfer front is also clearly affected by the flow rate. The experimental profiles (symbols) are compared to predictions of the dynamic adsorption model that was fitted to the outlet breakthrough
Fig. 5 Internal adsorption profiles during column breakthrough experiments; adsorption (left column) and desorption experiments (right column). Symbols are experimental data estimated according to Eq. 8 and solid lines are predictions from the dynamic column breakthrough model that uses parameters fitted to the conventional output. The color code indicates the time of the profiles relative to the residence time $\left(t_{r}=V_{\mathrm{b}} / f_{\text {in }}\right)$ (Color figure online)

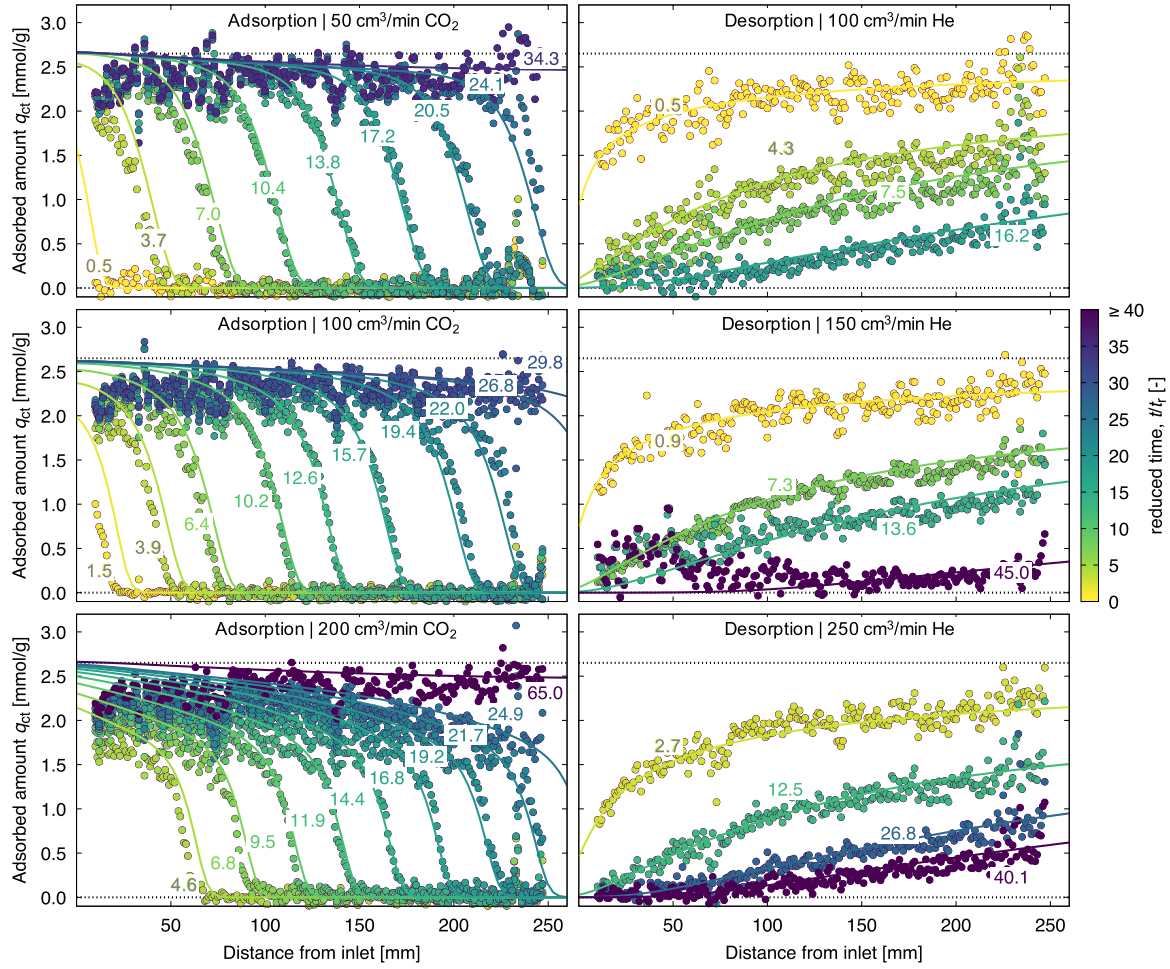


profiles (solid lines). There is an excellent agreement between experiments and simulations at each time, for the three flow rates and for both adsorption and desorption experiments. Slight deviations are observed in the early profiles during adsorption (up to approx. $7 \mathrm{~cm}$ from the inlet), which are likely due to the presence of the thermocouple that reduces the cross sectional area of the packed bed.

With increasing flow rate, the adsorption heat effects on the mass transfer zone become clearly visible and significant tailing appears at the trailing edge of the adsorption front. At the highest flow rate $\left(f_{\text {in }}=200 \mathrm{~cm}^{3} / \mathrm{min}\right)$, one can clearly distinguish each time-profile behind the front, while the same profiles are overlapping in the experiments at the lower flow rates $\left(f_{\text {in }}=50 \mathrm{~cm}^{3} / \mathrm{min}\right.$ and $\left.f_{\text {in }}=100 \mathrm{~cm}^{3} / \mathrm{min}\right)$. The experimental internal profiles provide for an added depth of information: a significant variation in the sliceaveraged adsorbed amount is present $\left(q_{\mathrm{ct}} \approx \pm 0.25 \mathrm{mmol} / \mathrm{g}\right)$, which cannot be explained by the measurement uncertainty $(< \pm 30 \mu \mathrm{mol} / \mathrm{g})$. Because the average bed density $\left(\rho_{\mathrm{b}}=0.435 \mathrm{~g} / \mathrm{cm}^{3}\right)$ was used to convert the X-ray CT measurement to an amount adsorbed per unit mass, these variations are likely due to heterogeneities that are inherent to random packings (see also Sect. 6). The results shown in Fig. 5 demonstrate the practical feasibility of applying X-ray CT to observe dynamic processes in commercial adsorbent systems. The quantitative agreement with the model further indicates that it should be possible to exploit this technique beyond the verification exercise and to reveal new insight on the dynamics of adsorption processes.

\subsection{Mixed gas feed}

To further verify the validity of the experimental protocol and the predictive ability of the numerical model, an experiment was conducted in which a feed stream containing 50\% $\mathrm{CO}_{2}$ and $50 \%$ He was injected at $f_{\text {in }}=100 \mathrm{~cm}^{3} / \mathrm{min}$ into the

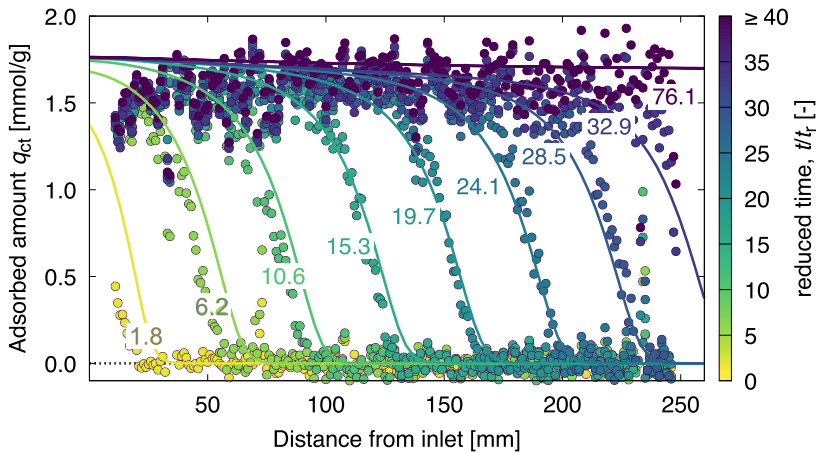

Fig. 7 Internal adsorption profiles of the adsorption breakthrough experiment carried out for a 50:50 $\mathrm{CO}_{2}: \mathrm{He}$ feed. Experimental profiles (symbols) are estimated according to Eq. 8, while the model prediction (solid lines) relies on parameters fitted to the $100 \mathrm{~cm}^{3} / \mathrm{min}$ experiment with a pure $\mathrm{CO}_{2}$ feed. The color code indicates the time of the profiles relative to the residence time $\left(t_{r}=V_{\mathrm{b}} / f_{\text {in }}\right)$ (Color figure online)

column initially regenerated $(y=0)$. Again, the analysis of the temporal evolution of the outlet composition, outlet flow rate, and the temperature at $7.2 \mathrm{~cm}$ from the inlet (Fig. 6, the "conventional" output), is complemented by direct observations on the progression of the mass transfer front within the adsorbent column obtained by X-ray CT (Fig. 7). In both figures, the experimental observations are compared to the results from the dynamic adsorption column model and an excellent agreement is observed for each data set. We note the characteristic decline in the temperature at about $1800 \mathrm{~s}$ after start of the experiment that is observed in both the measured and the simulated temperature profile. This behaviour is attributed to a feed with lower temperature than the column that reaches the thermocouple position in the absence of adsorption heat effects, i.e. once adsorption front has left the saturated column. We emphasize that the model has been used here in a predictive manner, i.e. no parameter
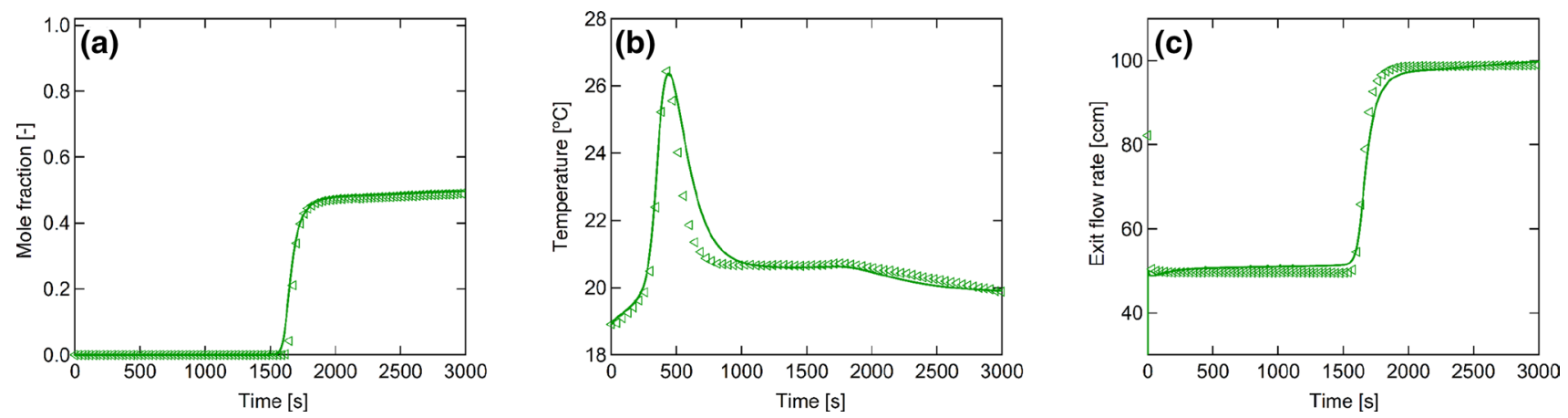

Fig. 6 Conventional output from the dynamic breakthrough experiments with mixed gas feed $\left(y_{\text {in }}=0.5\right)$ into the column initially regenerated $(y=0)$. Temporal evolution of a outlet composition, b internal temperature $(7.2 \mathrm{~cm}$ from inlet) and $\mathbf{c}$ outlet flow rate.
Symbols and lines correspond to experimental and simulation data, respectively. Experimental conditions: $p=100 \mathrm{kPa}, T=293.15 \mathrm{~K}$, $f_{\text {in }}=100 \mathrm{~cm}^{3} / \mathrm{min}$ 
fitting has taken place. The two heat transfer coefficients used to simulate this experiment are those obtained from the $\mathrm{CO}_{2}$ adsorption experiment carried out at $f_{\text {in }}=100 \mathrm{~cm}^{3} / \mathrm{min}$ and presented in Sect. 5.2 (values reported in Table 4).

There are two noticeable effects of feed composition on the propagation of the adsorption front. First, the breakthrough time, $t_{\mathrm{bt}}=1683 \mathrm{~s}(y=0.5)$ has increased compared to the value observed for the pure $\mathrm{CO}_{2}$ feed at the same total flow rate, $t_{\mathrm{bt}}=1232 \mathrm{~s}(y=1)$. Second, both outlet profiles (composition and flow rate) are more dispersed than their counterpart for the pure $\mathrm{CO}_{2}$ feed (Fig. 4a, c). These two observations are in line with the expected (1) concentration dependence of the breakthrough time with a non-linear adsorption isotherm and (2) greater axial dispersion as a result of interim pure helium flow before $\mathrm{CO}_{2}$ breakthrough. This can again be verified through the application of the equilibrium theory; the latter predicts an increase in the breakthrough time from $t_{\mathrm{bt}}^{*}=1353 \mathrm{~s}\left(y_{\text {in }}=1\right)$ to $t_{\mathrm{bt}}^{*}=1787 \mathrm{~s}\left(y_{\mathrm{in}}=0.5\right)$, and a substantial increase of the flow rate at the leading edge of the mass transfer zone $\left(f_{\text {out }}^{*}=1.6 \mathrm{~cm}^{3} / \mathrm{min}\right.$ at $y_{\text {in }}=1 \mathrm{vs} . f_{\text {out }}^{*}=50.6 \mathrm{~cm}^{3} / \mathrm{min}$ at $\left.y_{\text {in }}=0.5\right)$. As with the experiments using a pure $\mathrm{CO}_{2}$ feed, the breakthrough time predicted by the equilibrium theory is larger (6.2\%) than the value observed in Fig. 6, as a result of the adsorption heat effects that appear in the experiments.

\section{Discussion}

X-ray CT represents an exciting prospect for the study of adsorption dynamics in standard laboratory dynamic breakthrough systems. One of the benefits arising from the application of a tomographic technique for measuring adsorption is the ability to obtain information on experimental breakthrough curves within the bed and to make observations over multiple length-scales to access adsorption properties spatially. Several reasons justify the demand for this increase in the level of observational detail. First, the measurement of the variation in adsorbed amount in time and along one spatial dimension of the column underpins the design of robust processes for separations with stringent specifications, such as ultra-high purity products (Hosseinzadeh Hejazi et al. 2017). Second, the need to intensify current industrial pressure- temperature swing adsorption processes relies, among others, on the improvement of heat and mass transport through the design of contactor geometries that cannot be captured with traditional one-dimensional models (Claessens et al. 2020; Sharma et al. 2020; Wurzbacher et al. 2016). While three-dimensional modelling of complete pressure- (PSA), vacuum- (VSA), and temperature-swing adsorption (TSA) cycles is nowadays possible (Gautier et al. 2018; Lian et al. 2019; Qasem and Ben-Mansour 2018), these findings would need to be validated against direct measurements of time-resolved internal adsorbent loading profiles in three-dimensions. Third, the need of developing more realistic structural models of technical adsorbents requires understanding the effects that arise from variations in the local voidage properties within the adsorption column and within the individual porous particles. Because X-ray CT measurements contain three-dimensional data of the column, the analysis can be readily extended to higher resolution and smaller scales, a level of information that makes a first step towards bridging the gap between macroscopic process description and high-accuracy pore-scale measurements and theories.

We have presented above results from a series of breakthrough experiments using the model system $\mathrm{CO}_{2} /$ helium on activated carbon and successfully validated them against predictions from a one-dimensional dynamic adsorption column model. We present in the following the results obtained upon extracting from the same data set transient uptake profiles within a $1 \mathrm{~cm}$-thick section of the column, thereby extending the analysis to individual adsorbent pellets.

\subsection{Internal breakthrough curves}

The breakthrough profiles measured during the experiment carried out at $f_{\text {in }}=100 \mathrm{~cm}^{3} / \mathrm{min}$ using (a) pure $\left(p_{\mathrm{CO}_{2}}=100 \mathrm{kPa}\right)$ and (b) mixed gas feed $\left(p_{\mathrm{CO}_{2}}=50 \mathrm{kPa}\right)$ are plotted in Fig. 8 for ten slices within a $1 \mathrm{~cm}$-thick section of the column located at $7.4 \mathrm{~cm}$ from the inlet. As indicated in the inset of the figure, each curve is color-coded to represent each individual $1 \mathrm{~mm}$-thick slice. In agreement with the observations at the outlet of the column (Figs. 4 and 6), the internal profiles of the two experiments differ in the propagation velocity of the adsorption front and in the width of the front. The former results in a faster breakthrough for the experiment with the pure feed $\left(t_{\mathrm{bt}} \approx 290 \mathrm{~s}\right)$ as compared to the mixed feed $\left(t_{\mathrm{bt}} \approx 410 \mathrm{~s}\right)$. Closer inspection of the uptake rate of each individual profile also reveals a lag of about $50 \mathrm{~s}$ at half maximum between the upstream and downstream slice of the $1 \mathrm{~cm}$-long column section in both experiments. At the flow rate of the experiment, the equilibrium theory indicates that it would take 50-60 s for the shock wave to travel through the same volume, in agreement with our experimental observations. Notably, also the breakthrough times agree reasonably well with their counterpart estimated from the theory and using column-averaged properties $\left(\rho_{\mathrm{b}}=435 \mathrm{~kg} / \mathrm{m}^{3}, \epsilon=0.456\right)$, namely $t_{\mathrm{bt}}^{*}=350 \mathrm{~s}\left(q^{*}=2.61\right.$ $\mathrm{mol} / \mathrm{kg}$ at $\left.p_{\mathrm{CO}_{2}}=100 \mathrm{kPa}\right)$ and $t_{\mathrm{bt}}^{*}=460 \mathrm{~s}\left(q^{*}=1.73 \mathrm{~mol} /\right.$ $\mathrm{kg}$ at $\left.p_{\mathrm{CO}_{2}}=50 \mathrm{kPa}\right)$.

The experimental uptake profiles in both experiments are characterised by a substantial degree of asymmetry with a slower transition to the equilibrium loading at late times. For a system characterised by fast intra-particle mass transport (such as the one considered here), this effect is 

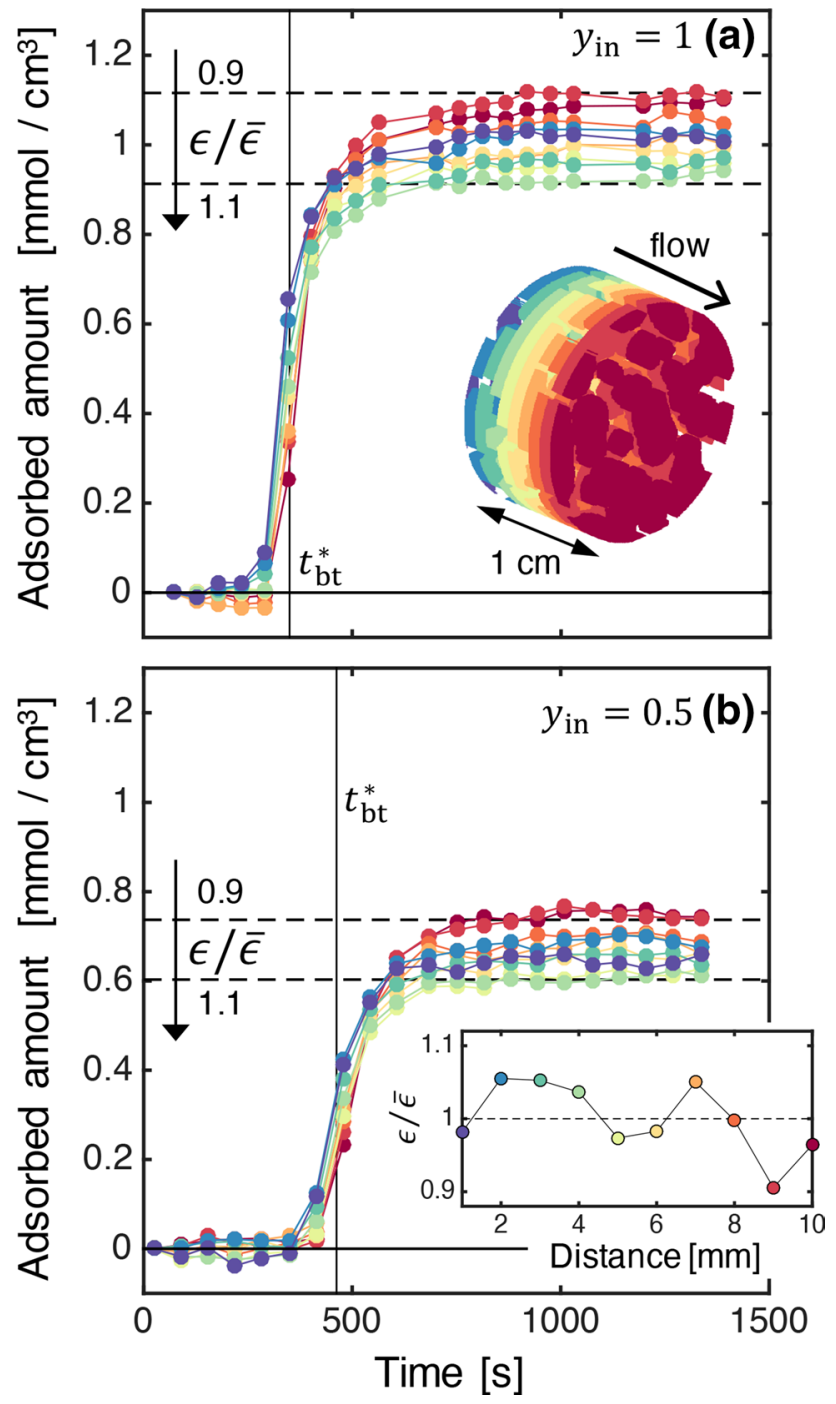

Fig. 8 Internal uptake adsorption curves measured by X-ray CT within a $1 \mathrm{~cm}$-thick section of the column located at $7.4 \mathrm{~cm}$ from the inlet. Curves are shown for ten $1 \mathrm{~mm}$-thick slices and their color indicates location (see inset in a). The vertical solid line represents the breakthrough time estimated by equilibrium theory, while the horizontal dashed lines indicate the expected variation in the adsorbed amount as a result of changes in the local bed porosity (see inset in b). a pure feed $\left(p_{\mathrm{CO}_{2}}=100 \mathrm{kPa}\right)$. b mixed gas feed $\left(p_{\mathrm{CO}_{2}}=50 \mathrm{kPa}\right)$. Total pressure: $p=100 \mathrm{kPa}$, temperature: $T=293.15 \mathrm{~K}$, flow rate: $f_{\text {in }}=100 \mathrm{~cm}^{3} / \mathrm{min}$

attributed to the non-isothermal nature of the uptake process. The experimental profiles also show smearing of the mass transfer zone that is more pronounced in the experiment with the mixed feed. This can be explained as the result of a larger axial dispersion, owing to the larger flow rate at the leading edge of the mass transfer zone (see Sect. 5.4). While one could also argue with a stronger selfsharpening effect present for the higher concentration feed, the equilibrium isotherm is only weakly non-linear and this effect is therefore unlikely to explain the marked difference in the uptake rate. However, this ability to reveal smearing of the mass transfer zone within the column, would enable exploring the self-sharpening effect in systems with higher degree of non-linearity as well as exploring more advanced ternary breakthrough experiments. The latter could be designed such that both shock and simple wave transitions are observed in a single adsorption breakthrough experiment.

The striking feature of the results shown in Fig. 8 is that the equilibrium amount adsorbed attained at late times differs among the ten slices. These variations are consistent with those observed in Figs. 5 and 7 across the whole column. Yet, the color coding used for the plotting (inset of Fig. 8a) further indicates that the observed trend is not random, but is consistent by and large between the two experiments. Notably, the ratio of the measured slices-averaged equilibrium capacity, $q_{\mathrm{ct}}(100 \mathrm{kPa}) / q_{\mathrm{ct}}(50 \mathrm{kPa})=1.50$, is in excellent agreement with its counterpart estimated from the adsorption isotherm $q^{*}(100 \mathrm{kPa}) / q^{*}(50 \mathrm{kPa})=1.51$. As indicated by the horizontal dashed lines, variations along the length of the column can be explained by local changes in the bed density to yield relative variations in the local bed porosity, $\epsilon / \bar{\epsilon}=0.9-1.1$ (inset of Fig. $8 \mathrm{~b}, \bar{\epsilon}$ is the average porosity of the $1-\mathrm{cm}$ long section). Yet, similar variations in the adsorbed amount can also be attributed to variations in the local average particle porosity, $\epsilon_{\mathrm{p}}=0.60-0.68$, at the measured column-averaged bed porosity $(\epsilon=0.456)$. Most likely, both effects will contribute to local variations in the adsorption properties of the column.

\subsection{Transient uptake of individual adsorbent pellets}

The analysis presented in the previous section is extended here to 12 individual pellets within the same $1 \mathrm{~cm}$-long section of the adsorption column. To this end, the raw X-ray $\mathrm{CT}$ tomograms were processed using the marker-controlled watershed segmentation algorithm described in Sect. S2. The separated adsorbent particles are shown in the bottom right corner of Fig. 9 and have been color-coded 1-12 to enable better visualisation. While the low resolution of the tomograms in the axial direction $(x, 1 \mathrm{~mm})$ limits the accuracy of the segmentation, the obtained result was deemed to be of sufficient quality for computing pellet-averaged quantities. The remaining panels in the figure show the transient uptake profiles for each individual pellet for the experiment carried out at $f_{\text {in }}=100 \mathrm{~cm}^{3} / \mathrm{min}$ using pure $\left(p_{\mathrm{CO}_{2}}=100 \mathrm{kPa}\right.$, circles) and mixed gas feed ( $p_{\mathrm{CO}_{2}}=50 \mathrm{kPa}$, triangles). The results mimic the behavior observed for slice- and columnaveraged properties discussed in the previous sections and show the influence of feed composition on the shape of the uptake curve. Specifically, for each pellet, two curves are obtained that are characterised by a distinct uptake rate and and a distinct equilibrium loading at late times. The latter 

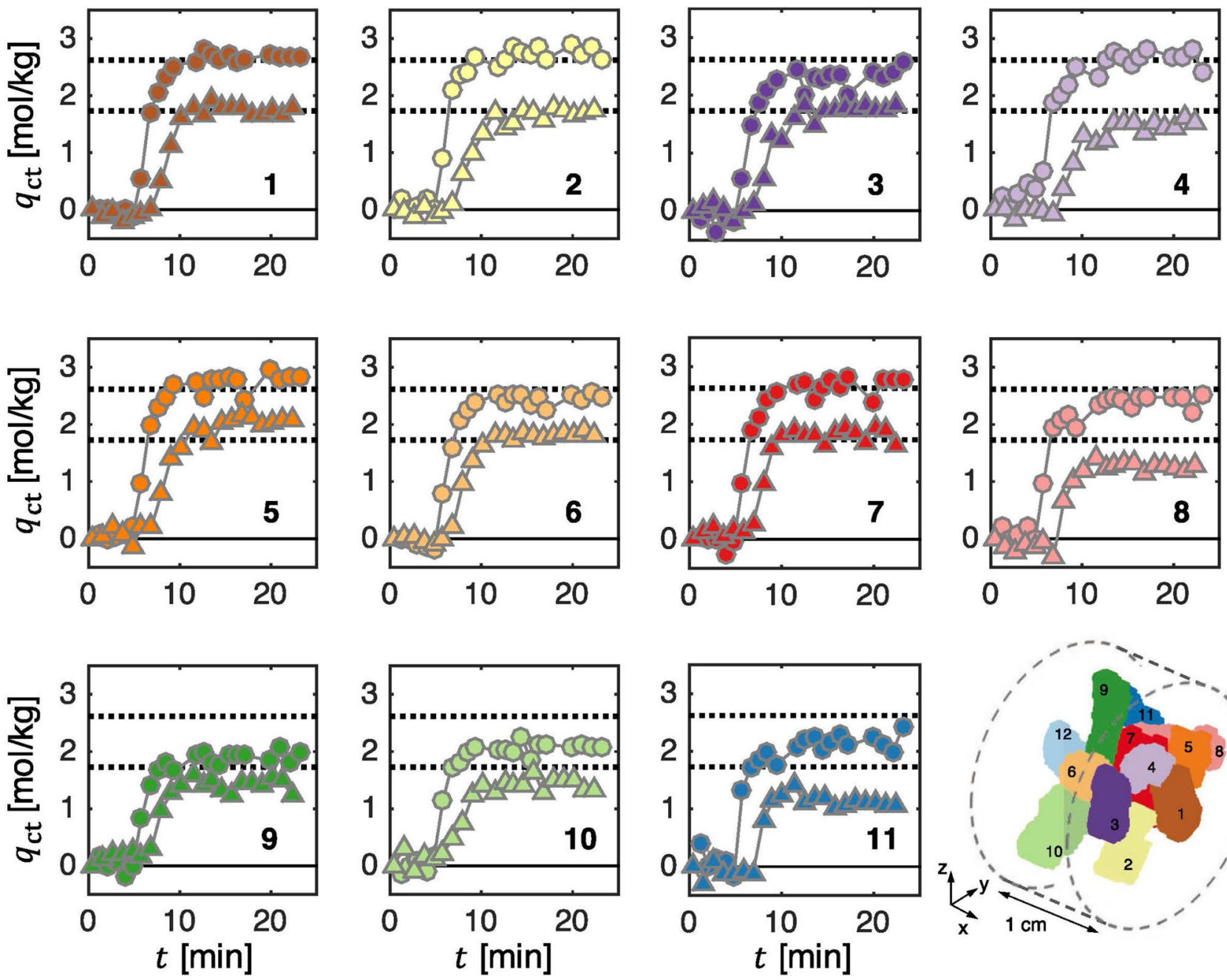

Fig. 9 Uptake adsorption curves measured by X-ray CT for individual adsorbent pellets within a $1 \mathrm{~cm}$-thick section of the column located at $7.4 \mathrm{~cm}$ from the inlet. Curves are color-coded 1-12 to represent each pellet. Circles and triangles refer to data obtained from the pure $\left(p_{\mathrm{CO}_{2}}=100 \mathrm{kPa}\right)$ and mixed feed experiment $\left(p_{\mathrm{CO}_{2}}=50 \mathrm{kPa}\right)$,

agree by and large with results obtained independently by volumetry (Fig. 3), as indicated by the horizontal dashed lines for the two partial pressures considered here. The ability to monitor simultaneously uptake curves on individual pellets by X-ray CT complements the output of microscopic diffusion experiments via NMR tracer desorption and NMR PFG self-diffusion measurements (Kärger et al. 2012). As demonstrated in that study, even for commercial zeolite samples, there are considerable variations between different batches of product and between different pellets from the same batch, owing to e.g., differences in hydrothermal stability.

The individual isotherm plots extracted from the equilibrium loadings at late times are shown in Fig. 10 and confirm the reliability of the X-ray CT measurement at the scale of

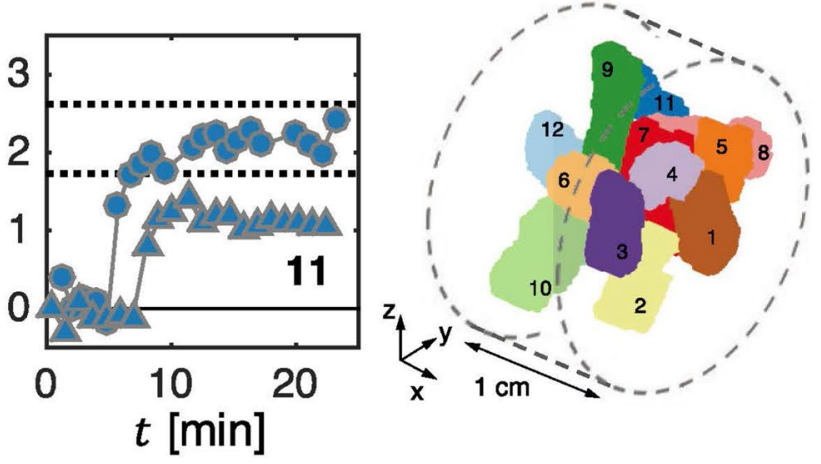

respectively. The horizontal dashed lines indicate the corresponding equilibrium amount adsorbed measured by the volumetry. Total pressure: $p=100 \mathrm{kPa}$, temperature: $T=293.15 \mathrm{~K}$, flow rate: $f_{\text {in }}=100 \mathrm{~cm}^{3} \mathrm{~min}^{-1}$

an individual pellet (the dashed lines are the dual-site Langmuir model isotherms fitted onto the volumetry data). It can be seen that while the majority of the pellets conform to the expected column-averaged loading, local deviations in the uptake capacity are observed (pellets 5, 8, 9, 10, 11). Because a unique average particle density was used to convert the X-ray CT measurement to an amount adsorbed per unit mass $\left(\rho_{\mathrm{p}}=500 \mathrm{~kg} / \mathrm{m}^{3}\right)$, this behaviour is likely to ensue from variations in the intraparticle porosity, as anticipated in the previous section. This result further indicates that for the system considered in this study, local variations in the bed density drive the observed variability in the adsorbed amount at the column-scale (Figs. 5, 7 and 8) and that the (more subtle) variations in the pellet porosity are subordinate to it. 

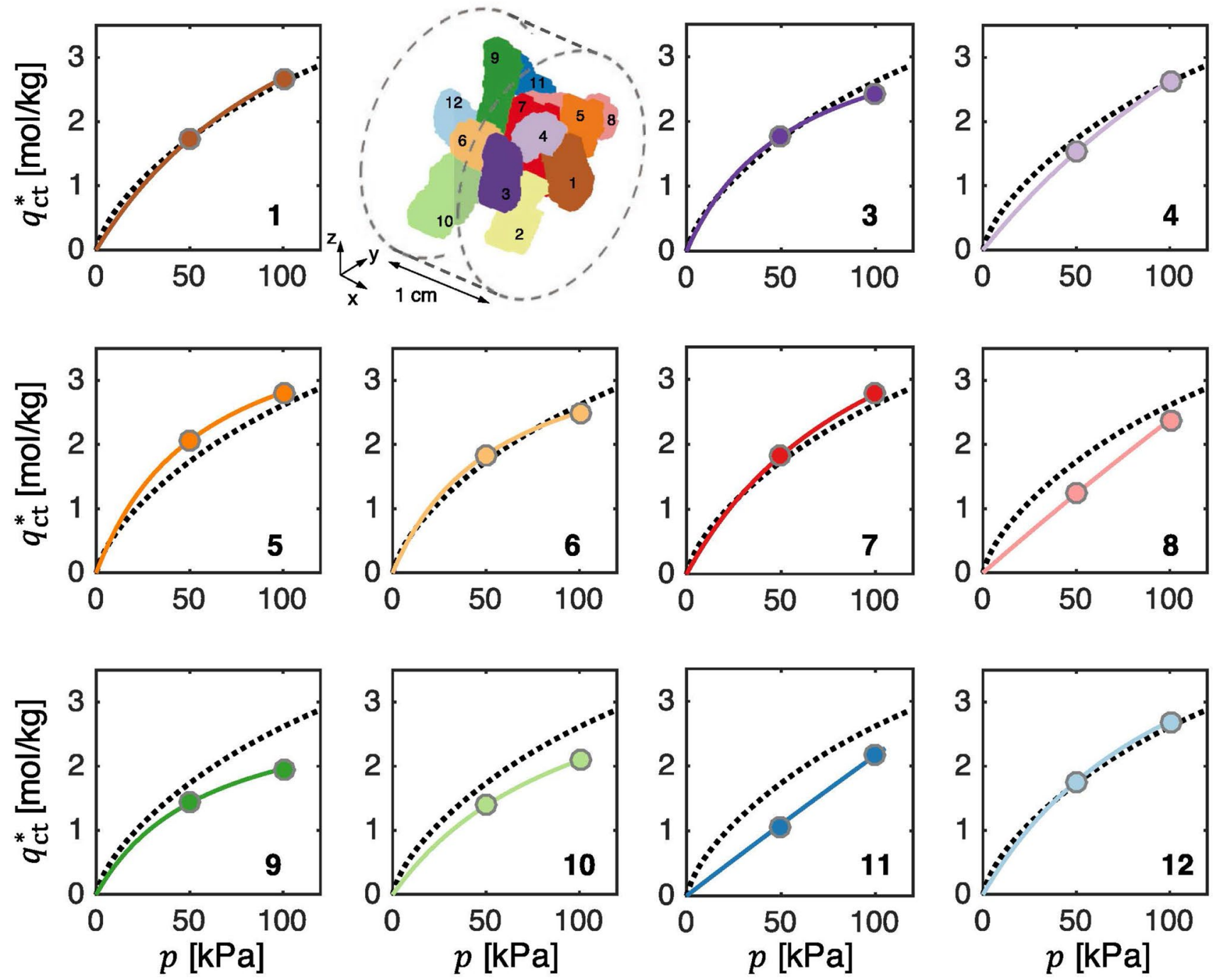

Fig. 10 Adsorption isotherms measured by X-ray CT on 12 pellets within a $1 \mathrm{~cm}$-thick section of the column located at $7.4 \mathrm{~cm}$ from the inlet (same experimental conditions and color-coding as in Fig. 9). The colored lines are fits obtained upon application of a single-site

Langmuir adsorption model to each data set, while the dotted black line is the adsorption isotherm measured by the volumetry (Fig. 3) (Color figure online)

This novel ability to monitor each pellet individually further suggests that it should be possible to estimate additional parameters, such as the effective diffusivity of the adsorptive in the adsorbent particles with a slight modification of the experiment design. Most importantly, the ability to move seamlessly from the column- down to the pellet-scale within a single experiment is unique to X-ray CT and highlights its potential as a tool for studying adsorption or reaction systems involving microporous sorbents or catalysts under realistic conditions.

\section{Conclusions}

This study has demonstrated the practical feasibility of applying X-ray CT to measure transient gas uptake profiles in adsorbent systems containing a commercial activated carbon adsorbent on the scale from a packed adsorbent column down to an individual adsorbent particle. A "simple" adsorptive was considered, $\mathrm{CO}_{2}$ /helium, in both adsorption and desorption mode, to (i) demonstrate the feasibility of the approach without the need of a strongly attenuating agent and (ii) to verify conformity of the measured profiles to theoretical expectations. To this end, a generic one-dimensional adsorption column model was successfully applied to describe the breakthrough experiments, including both 
outlet and internal transient profiles of concentration and temperature. The results illustrate the rich variety of propagation behavior of the mass transfer zone depending on the feed composition and the flow rate. The ability to measure such profiles directly and down to the the scale of a single pellet provides an exciting prospect for future studies. In fact, the X-ray CT imagery contain 3D data of the column that can be used, alongside numerical tools, to bridge the gap between macroscopic process description and high-accuracy pore-scale measurements and theories.

Open Access This article is licensed under a Creative Commons Attribution 4.0 International License, which permits use, sharing, adaptation, distribution and reproduction in any medium or format, as long as you give appropriate credit to the original author(s) and the source, provide a link to the Creative Commons licence, and indicate if changes were made. The images or other third party material in this article are included in the article's Creative Commons licence, unless indicated otherwise in a credit line to the material. If material is not included in the article's Creative Commons licence and your intended use is not permitted by statutory regulation or exceeds the permitted use, you will need to obtain permission directly from the copyright holder. To view a copy of this licence, visit http://creativecommons.org/licenses/by/4.0/.

\section{References}

Anderson, B.G., Gauw, F.J.M.M., Noordhoek, N.J., van Ijzendoorn, L.J., van Santen, R.A., Voigt, M.J.A.: Mass transfer of alkanes in zeolite packed-bed reactors studied with positron emission profiling (PEP). 1. Experiments. Ind. Eng. Chem. Res. 37(3), 815-824 (1998)

Ansari, H., Joss, L., Hwang, J., Trusler, M., Maitland, G., Pini, R.: Supercritical adsorption in micro- and meso-porous carbons and its utilisation for textural characterisation. Microporous Mesoporous Mater. 308, 110537 (2020)

Bär, N.K., Balcom, B.J., Ruthven, D.M.: Direct measurement of transient concentration profiles in adsorbent particles and chromatographic columns by MRI. Ind. Eng. Chem. Res. 41(9), 2320-2329 (2002)

Cheah, K.Y., Chiaranussati, N., Hollewand, M.P., Gladden, L.F.: Coke profiles in deactivated alumina pellets studied by NMR imaging. Appl. Catal. A 115(1), 147-155 (1994)

Cheng, Y., Huang, Q., Eić, M., Balcom, B.J.: $\mathrm{CO}_{2}$ dynamic adsorption/desorption on zeolite $5 \mathrm{~A}$ studied by $13 \mathrm{C}$ magnetic resonance imaging. Langmuir 21(10), 4376-4381 (2005)

Claessens, B., Dubois, N., Lefevere, J., Mullens, S., Cousin-SaintRemi, J., Denayer, J.F.M.: 3D-printed ZIF8 monoliths for biobutanol recovery. Ind. Eng. Chem. Res. 59, 8813-8824 (2020)

DeWitt, S.J.A., Sinha, A., Kalyanaraman, J., Zhang, F., Realff, M.J., Lively, R.P.: Critical comparison of structured contactors for adsorption-based gas separations. Annu. Rev. Chem. Biomol. Eng. 9, 129-152 (2018)

Dubinin, M.M., Erashko, I.T., Kadlec, O., Ulin, V.I., Voloshchuk, A.M., Zolotarev, P.P.: Kinetics of physical adsorption by carbonaceous adsorbents of biporous structure. Carbon 13(3), 193-200 (1975)

Gautier, R., Dbouk, T., Campesi, M.A., Hamon, L., Harion, J.L., Pré, P.: Pressure-swing-adsorption of gaseous mixture in isotropic porous medium: Transient 3D modeling and validation. Chem. Eng. J. 348, 1049-1062 (2018)
Haghpanah, R., Majumder, A., Nilam, R., Rajendran, A., Farooq, S., Karimi, I.A., Amanullah, M.: Multiobjective optimization of a four-step adsorption process for postcombustion $\mathrm{CO}_{2}$ capture via finite volume simulation. Ind. Eng. Chem. Res. 52(11), 4249 4265 (2013)

Hosseinzadeh Hejazi, S.A., Rajendran, A., Sawada, J.A., Kuznicki, S.M.: Dynamic column breakthrough and process studies of highpurity oxygen production using silver-exchanged titanosilicates. Ind. Eng. Chem. Res. 55(20), 5993-6005 (2016)

Hosseinzadeh Hejazi, S.A., Estupiñan Perez, L., Rajendran, A., Kuznicki, S.: Cycle development and process optimization of high-purity oxygen production using silver-exchanged titanosilicates. Ind. Eng. Chem. Res. 56(19), 5679-5691 (2017)

Johnson, T.F., Levison, P.R., Shearing, P.R., Bracewell, D.G.: X-ray computed tomography of packed bed chromatography columns for three dimensional imaging and analysis. J. Chromatogr. A 1487, 108-115 (2017)

Joss, L., Pini, R.: Digital adsorption: 3D imaging of gas adsorption isotherms by X-ray computed tomography. J. Phys. Chem. C 121(48), 26903-26915 (2017)

Joss, L., Pini, R.: 3D mapping of gas physisorption for the spatial characterisation of nanoporous materials. ChemPhysChem 20(4), 524-528 (2019)

Karacan, C.O., Okandan, E.: Adsorption and gas transport in coal microstructure: investigation and evaluation by quantitative $\mathrm{X}$-ray CT imaging. Fuel 80(4), 509-520 (2001)

Kärger, J., Ruthven, D.M., Theodorou, D.N.: Diffusion in Nanoporous Materials. Wiley, Hoboken (2012)

Kayser, J.C., Knaebel, K.S.: Pressure swing adsorption: development of an equilibrium theory for binary gas mixtures with nonlinear isotherms. Chem. Eng. Sci. 44(1), 1-8 (1989)

Knox James, C., Ebner, A.D., LeVan, M.D., Cocker, R.K., Ritter, J.A.: Limitations of breakthrough curve analysis in fixed-bed adsorption. Ind. Eng. Chem. Res. 55, 4734-4748 (2016)

Kwapinski, W., Winterberg, M., Tsotsas, E., Mewes, D.: Modeling of the wall effect in packed bed adsorption. Chem. Eng. Technol. 27(11), 1179-1186 (2004)

Lian, Y., Deng, S., Li, S., Guo, Z., Zhao, L., Yuan, X.: Numerical analysis on $\mathrm{CO}_{2}$ capture process of temperature swing adsorption (TSA): optimization of reactor geometry. Int. J. Greenhouse Gas Control 85, 187-198 (2019)

Lodewyckx, P., Blacher, S., Léonard, A.: Use of X-ray microtomography to visualise dynamic adsorption of organic vapour and water vapour on activated carbon. Adsorption 12(1), 19-26 (2006)

Lottaz, A., Lavanchy, A.: X-ray computer-tomography, a new experimental method for the study of dynamic sorption processes on activated carbon. Carbon 25(4), 533-539 (1987)

Lu, X., Miao, P., Watson, A.T., Pepin, G.P., Moss, R.M., Semmelbeck, M.: X-ray computed tomography studies of gas storage and transport in Devonian shales. AIChE J. 40(7), 1246-1253 (1994)

Maire, E., Whiters, J.: Quantitative X-ray tomography. Int. Mater. Rev. 59, 1-43 (2014)

Martin, A.J., Mitchell, S., Kunze, K., Weston, K.C., Pérez-Ramírez, J.: Visualising compositional heterogeneity during the scale up of multicomponent zeolite bodies. Mater. Horizons 4(5), 857-861 (2017)

Mayo, S., Josh, M., Nesterets, Y., Esteban, L., Pervukhina, M., Clennell, M.B., Maksimenko, A., Hall, C.: Quantitative micro-porosity characterization using synchrotron micro-ct and xenon k-edge subtraction in sandstones, carbonates, shales and coal. Fuel 154, 167-173 (2015)

Mayo, S., Josh, M., Kasperczyk, D., Kear, J., Zhang, J., Dautriat, J., Pervukhina, M., Clennell, M.B., Sakurovs, R., Sherwood, N.: Dynamic micro-CT study of gas uptake in coal using $\mathrm{Xe}, \mathrm{Kr}$ and $\mathrm{CO}_{2}$. Fuel 212, 140-150 (2018) 
Mazzotti, M., Rajendran, A.: Equilibrium theory-based analysis of nonlinear waves in separation processes. Annu. Rev. Chem. Biomol. Eng. 4, 119-141 (2013)

Nepryahin, A., Holt, E.M., Fletcher, R.S., Rigby, S.P.: Structuretransport relationships in disordered solids using integrated rate of gas sorption and mercury porosimetry. Chem. Eng. Sci. 152, 663-673 (2016)

Noordhoek, N.J., van Iizendoorn, L.J., Anderson, B.G., de Gauw, F.J., van Santen, R.A., de Voigt, M.J.: Mass transfer of alkanes in zeolite packed-bed reactors studied with positron emission profiling. 2. Modeling. Ind. Eng. Chem. Res. 37(3), 825-833 (1998)

Pavlovskaya, G., Six, J., Meersman, T., Gopinathan, N., Rigby, S.P.: NMR imaging of low pressure, gas-phase transport in packed beds using hyperpolarized Xenon-129. AIChE J. 61, 4013-4019 (2015)

Pini, R.: Multidimensional quantitative imaging of gas adsorption in nanoporous solids. Langmuir 30(37), 10984-10989 (2014)

Pini, R., Joss, L.: See the unseen: applications of imaging techniques to study adsorption in microporous materials. Curr. Opin. Chem. Eng. 24, 37-44 (2019)

Qasem, N.A.A., Ben-Mansour, R.: Energy and productivity efficient vacuum pressure swing adsorption process to separate $\mathrm{CO}_{2}$ from $\mathrm{CO}_{2} / \mathrm{N}_{2}$ mixture using mg-MOF-74: A CFD simulation. Appl. Energy 209, 190-202 (2018)

Rufford, T.E., Watson, G.C.Y., Saleman, T.L., Hofman, P.S., Jensen, N.K., May, E.F.: Adsorption equilibria and kinetics of methane + nitrogen mixtures on the activated carbon Norit RB3. Ind. Eng. Chem. Res. 52(39), 14270-14281 (2013)
Ruthven, D.M.: Principles of Adsorption and Adsorption Processes. Wiley, New York (1984)

Sharma, I., Mennitto, R., Friedrich, D., Brandani, S.: Combining the nonuniform structure and flow maldistribution for the accurate prediction of the process performance of monolithic adsorbent systems. Ind. Eng. Chem. Res. 59, 3162-3172 (2020)

Sircar, S., Golden, T.C.: Purification of hydrogen by pressure swing adsorption. Sep. Sci. Technol. 35, 667-687 (2006)

Vega, B., Dutta, A., Kovscek, A.R.: CT imaging of low-permeability, dual-porosity systems using high X-ray contrast gas. Transp. Porous Media 101(1), 81-97 (2014)

Vinegar, H.J., Wellington, S.L.: Tomographic imaging of threephase flow experiments. Rev. Sci. Instrum. 58(1), 96-107 (1987)

Watson, A.T., Mudra, J.: Characterization of Devonian shales with X-ray-computed tomography. SPE Formation Eval. 9(03), 209_ $212(1994)$

Wellington, S.L., Vinegar, H.J.: X-ray computerized tomography. J. Petrol. Technol. 39(08), 885-898 (1987)

Withjack, E.: Computed tomography for rock-property determination and fluid-flow visualization. SPE Form. Eval. 3(04), 696-704 (1988)

Wurzbacher, J.A., Gebald, C., Samuel, B., Steinfeld, A.: Heat and mass transfer of temperature-vacuum swing desorptionfor $\mathrm{CO}_{2}$ capture from air. Chem. Eng. J. 283, 1329-1338 (2016)

Publisher's Note Springer Nature remains neutral with regard to jurisdictional claims in published maps and institutional affiliations. 